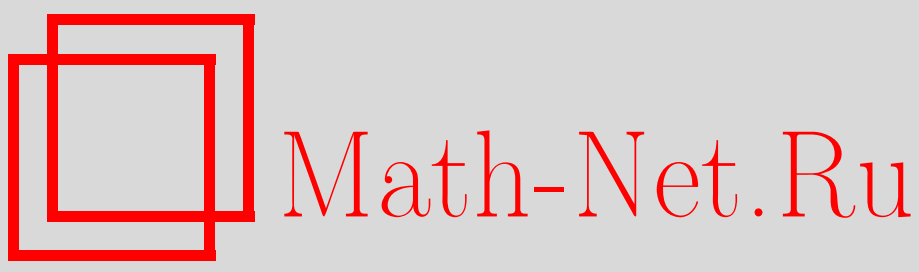

М. И. Дьяченко, Сферические частичные суммы двойных рядов Фурье функций с ограниченной обобщенной вариацией, Матем. сб., 1997, том 188, номер 1, 29-58

DOI: https://doi.org/10.4213/sm186

Использование Общероссийского математического портала Math-Net.Ru подразумевает, что вы прочитали и согласны с пользовательским соглашением http: //www. mathnet.ru/rus/agreement

Параметры загрузки:

IP: 35.173 .219 .149

26 апреля 2023 г., 13:52:08 
УДК 517.52

\author{
М. И. Дьяченко
}

\title{
Сферические частичные суммы двойных рядов Фурье функций с ограниченной обобщенной вариацией
}

В работе изучаются сферические частичные суммы двойных рядов Фурье функций из классов Ватермана. Основным результатом статьи является нижеследующий.

Теорема 1. Пусть некоторое $\varepsilon>0$ и последовательность $\Lambda_{\varepsilon}=$

$$
\begin{gathered}
\left\{\frac{n^{3 / 4}}{(\ln (n+1))^{1 / 2+\varepsilon}}\right\}_{n=1}^{\infty} . \text { Tогда, если функиия } f(x, y) \in \Lambda_{\varepsilon} B V\left(T^{2}\right), a \\
I_{r}(f)=\sup _{x, y \in T} \sup _{u, v \in[-1,1]} J_{r}(f) \\
=\sup _{x, y \in T} \sup _{u, v \in[-1,1]} \sum_{r-1<|(m, n)| \leqslant r+1} \mid a_{m, n}\left(\psi_{x, y, u, v} \mid \leqslant C\right.
\end{gathered}
$$

npu $r \geqslant 1$, əдe

$\psi_{x, y, u, v}(s, t)=\psi(s, t)=f(x+t, y+s) w(t) w(s) e^{-i(t u+s v)}, \quad w(\tau)=\frac{\tau}{2 \sin (\theta / 2)}$

то при любом $R \geqslant 1$ выполняется неравенство

$$
\sup _{R \geqslant 1(x, y) \in T^{2}} \sup _{R}\left|S_{R}(f, x, y)\right| \leqslant C(f, \varepsilon) .
$$

Рассматриваются также вопросы сходимости по кругам рядов Фурье характеристических функций выпуклых множеств на плоскости.

Библиографй: 12 названий.

\section{§1. Введение}

Целью настоящей статьи является получение ряда новых результатов о поведении сферических сумм двойных рядов Фурье функций из классов Ватермана [1]. Предварительно введем некоторые обозначения.

Пусть $\mathbb{Z}$ - это множество всех целых чисел, $T^{2}=[-\pi, \pi)^{2}$. Все рассматриваемые ниже функции будут предполагаться $2 \pi$-периодическими по каждой переменной. Для функций $f(x, y) \in L\left(T^{2}\right)$ через

$$
\sum_{m, n \in \mathbb{Z}} a_{m, n} e^{i(m x+n y)}
$$

Работа выполнена при поддержке Международного научного фонда (грант № NCJ 300) и Российского фонда фундаментальных исследований (грант № 96-01-00094). 
будет обозначаться их двойной ряд Фурье, а через

$$
S_{r}(f, x, y)=\sum_{m, n \in \mathbb{Z},|(m, n)| \leqslant r} a_{m, n} e^{i(m x+n y)},
$$

где $r \geqslant 1,-$ частичные сферические суммы этого ряда.

Если $1 \leqslant p \leqslant \infty$ и $f(x, y) \in L_{p}\left(T^{2}\right)\left(L_{\infty}\left(T^{2}\right) \equiv C\left(T^{2}\right)\right)$, то через $\omega\left(f, \delta_{1}, \delta_{2}\right)_{p}$, $\omega_{1}\left(f, \delta_{1}\right)_{p}$ и $\omega_{2}\left(f, \delta_{2}\right)_{p}$, где $\delta_{1}, \delta_{2}>0$, будут обозначаться смешанный и частные модули непрерьвности функции $f(x, y)$ в пространстве $L_{p}\left(T^{2}\right)$, соответственно, т.е., например,

$\omega\left(f, \delta_{1}, \delta_{2}\right)_{p}=\sup _{|t| \leqslant \delta_{1},|h| \leqslant \delta_{2}}\|f(x, y)-f(x+t, y)-f(x, y+h)+f(x+t, y+h)\|_{L_{p}\left(T^{2}\right)}$.

Нам также понадобится определение классов Ватермана $\Lambda B V\left(T^{2}\right)$. Вначале определим соответствующий одномерный класс.

ОПРЕДЕЛЕНИЕ 1 . Пусть последовательность $\Lambda=\left\{\lambda_{j}\right\}_{j=1}^{\infty} \in \Phi$, т.е. $\Lambda$ является монотонно неубьвающей последовательностью положительных чисел такой, что

$$
\sum_{j=1}^{\infty} \lambda_{j}^{-1}=\infty
$$

и $f(x)-2 \pi$-периодическая функция. Тогда говорят, что $f(x)$ принадлежит классу Ватермана $\Lambda B V(T)$, если

$$
\Lambda V_{T} f(x)=\sup _{a \in \mathbb{R}} \sup _{\Gamma} \sum_{k=1}^{n} \frac{\left|f\left(I_{k}\right)\right|}{\lambda_{k}} \equiv \sup _{a \in \mathbb{R}} \sup _{\Gamma} \sum_{k=1}^{n} \frac{\left|f\left(\beta_{k}\right)-f\left(\alpha_{k}\right)\right|}{\lambda_{k}}<\infty,
$$

где $\Gamma$ - система неперекрывающихся интервалов $\left\{I_{k}=\left(\alpha_{k}, \beta_{k}\right)\right\}_{k=1}^{n}$ из $T+a=$ $[-\pi+a, \pi+a)$.

В двумерном случае соответствуюшее определение таково.

ОПРЕДЕЛЕНИЕ 2. Пусть последовательность $\Lambda \in \Phi$ и $f(x, y)$ является $2 \pi$-периодической по каждой переменной и измеримой на $T^{2}$ функцией. Тогда говорят, что $f(x, y) \in \Lambda B V\left(T^{2}\right)$ в том и только в том случае, когда:

1. ограничения $f(x,-\pi), f(-\pi, y) \in \Lambda B V(T)$;

2. величина

$$
\Lambda V_{T^{2}} f(x, y)=\sup _{(a, b) \in \mathbb{R}^{2}} \sup _{\Gamma_{1}, \Gamma_{2}} \sum_{k=1}^{n} \sum_{r=1}^{m} \frac{\left|f\left(I_{k} \times J_{r}\right)\right|}{\lambda_{k} \lambda_{r}}<\infty,
$$

где

$$
f\left(I_{k} \times J_{r}\right)=f\left(\alpha_{k}, \gamma_{r}\right)-f\left(\alpha_{k}, \delta_{r}\right)-f\left(\beta_{k}, \gamma_{r}\right)+f\left(\beta_{k}, \delta_{r}\right)
$$

для всех $k$ и $r$, а $\Gamma_{1}$ и $\Gamma_{2}-$ суть две системы неперекрываюшихся интервалов $\left\{I_{k}=\left(\alpha_{k}, \beta_{k}\right)\right\}_{k=1}^{n}$ и $\left\{J_{r}=\left(\gamma_{r}, \delta_{r}\right)\right\}_{r=1}^{m}$ из $T+a$ и $T+b$, соответственно. Если $\Lambda \equiv 1$, то соответствующий класс обозначаем $B V\left(T^{2}\right)$. 
Отметим, что поскольку любая функция из класса Ватермана очевидно ограничена, для $f(x, y) \in \Lambda B V\left(T^{2}\right)$ можно определить величину

$$
\Lambda V f=\Lambda V_{T^{2}} f(x, y)+\Lambda V_{T} f(-\pi, y)+\Lambda V_{T} f(x,-\pi)+\sup _{T^{2}}|f(x, y)| .
$$

Всюду ниже через $C$ будут обозначаться положительные абсолютные постоянные (не обязательно одинаковые в различных случаях), через $C(f)$ - положительные постоянные, зависящие лишш от функции $f(x, y)$ и т. д.

Сферические суммы рядов Фурье функций, имеющих ограниченную в том или ином смысле вариацию, изучались рядом авторов. Так, К. Чандрасекхаран и С. Минакшисундарам [2] установили, что если $f(x, y)=\varphi(x) g(y)$, где функции $\varphi(x), g(y)$ имеют ограниченную вариацию, то сферические частичные суммы ее ряда Фурье сходятся всюду. Б.И. Голубов [3] доказал сходимость всюду средних Бохнера-Рисса критического порядка рядов Фурье функций из несколько более широкого класса, чем $B V\left(T^{2}\right)$. Автором [4] было показано, что если функция $f(x, y) \in B V\left(T^{2}\right)$, то, в частности, ее ряд Фурье сферически сходится всюду. Им же [5] установлено, что сферические частичные суммы рядов Фурье функций из класса $\Lambda_{0} B V\left(T^{2}\right)$, где $\Lambda_{0} \equiv\{\sqrt{n}\}_{n=1}^{\infty}$ равномерно ограничены постоянной, зависящей лишш от величины $\Lambda_{0} V f$. При этом, если $f(x, y) \in \Lambda B V\left(T^{2}\right)$, где $\lambda_{j}=o(\sqrt{j})$ при $j \rightarrow \infty$, то ряд Фурье функции $f(x, y)$ сферически сходится всюду.

Отметим, что основным методом исследования в данной статье является разработанный В.А. Юдиным [6] метод замены сумм $S_{r}(f, x, y)$ на

$$
S_{r}^{\prime}(f, x, y)=\sum_{m, n \in \mathbb{Z}} a_{m, n}^{\prime} e^{i(m x+n y)}
$$

где $a_{m, n}^{\prime}=a_{m, n}$, если $I_{m, n}=\left[m-\frac{1}{2}, m+\frac{1}{2}\right] \times\left[n-\frac{1}{2}, n+\frac{1}{2}\right] \subset B_{r}=\{(x, y):$ $|(x, y)| \leqslant r\}$, и

$$
a_{m, n}^{\prime}=a_{m, n}^{\prime}(r, x, y)=\frac{1}{4 \pi^{2}} \int_{T+x} \int_{T+y} f(t, s) \frac{\xi_{x, y}\left(I_{m, n} \cap B_{r}\right)}{\xi_{x, y}\left(I_{m, n}\right)} e^{-i(m t+n s)} d t d s
$$

в противном случае, где $\xi_{x, y}(E)=\iint_{E} e^{-i(t-x) u} e^{-i(s-y) v} d u d v$.

Во втором параграфе будут изучаться свойства модифицированных ядер Дирихле $D_{r}^{\prime}(x, y)$ и интегралов от таких ядер, где

$$
S_{r}^{\prime}(f, x, y)=\frac{1}{\pi^{2}} \iint_{T^{2}} f(x+t, y+s) D_{r}^{\prime}(t, s) d t d s .
$$

Основньми результатами этого параграфа являются нижеследующие.

ПРЕДЛОЖЕНИЕ 1 . Пусть $(u, v) \in(0, \pi)^{2}$. Тогда при любом $R \geqslant 1$ справедливы следующие неравенства

$$
\left|\int_{u}^{\pi} \int_{v}^{\pi} D_{R}^{\prime}(x, y) d x d y\right| \leqslant \frac{C}{R \max (u, v)+1}
$$

$u$

$$
\left|\int_{u}^{\pi} \int_{v}^{\pi} D_{R}^{\prime}(x, y) d x d y\right| \leqslant \frac{C}{(R \max (u, v)+1)^{1 / 2}(R \min (u, v)+1)} .
$$


ПРЕДЛОЖЕНИЕ 2. Существуют такие положсительные постоянные $C_{1}-C_{6}$, что при любом $R \geqslant C_{1}$ и при любьх $u \in\left[C_{2} / \sqrt{R}, C_{3}\right], v \in\left[C_{4} u, C_{5}\right]$ таких, что $|\sin (\rho-\pi / 4)|>1 / 5$, әде $\rho=R \sqrt{u^{2}+v^{2}}$, справедливо неравенство

$$
\left|\int_{u}^{\pi} \int_{v}^{\pi} D_{R}^{\prime}(x, y) d x d y\right| \geqslant \frac{C_{6}}{R^{3 / 2} u \sqrt{v}} .
$$

Из предложения 2 видно, что оценка (1) не может быть улучшена сразу для всех $R, u$ и $v$.

Третий параграф статьи посвящен приложению результатов $\S 2$ к изучению поведения сферических частичных сумм рядов Фурье функций двух переменных из классов Ватермана. Основньми результатами здесь являются нижеследующие.

ТЕОРема 1. Пусть некоторое $\varepsilon>0$ и последовательность

$$
\Lambda_{\varepsilon}=\left\{\frac{n^{3 / 4}}{(\ln (n+1))^{1 / 2+\varepsilon}}\right\}_{n=1}^{\infty} .
$$

Тогда, если функиия $f(x, y) \in \Lambda_{\varepsilon} B V\left(T^{2}\right)$, a

$$
I_{r}(f)=\sup _{x, y \in T} \sup _{u, v \in[-1,1]} J_{r}(f)=\sup _{x, y \in T} \sup _{u, v \in[-1,1]} \sum_{r-1<|(m, n)| \leqslant r+1}\left|a_{m, n}\left(\psi_{x, y, u, v}\right)\right|
$$

npu $r \geqslant 1$, əдe

$\psi_{x, y, u, v}(s, t)=\psi(s, t)=f(x+t, y+s) w(t) w(s) e^{-i(t u+s v)}, \quad w(\tau)=\frac{\tau}{2 \sin (\tau / 2)}$,

то при любом $R \geqslant 1$ выполняется неравенство

$$
\sup _{(x, y) \in T^{2}}\left|S_{R}(f, x, y)\right| \leqslant C I_{R+1}(f)+C(\varepsilon) \Lambda_{\varepsilon} V f .
$$

Отсюда, если

$$
I_{r}(f) \leqslant C
$$

при всех $r$, mo

$$
\sup _{R \geqslant 1} \sup _{(x, y) \in T^{2}}\left|S_{R}(f, x, y)\right| \leqslant C(f, \varepsilon) .
$$

В частности, если $\Lambda_{0} \equiv\{\sqrt{n}\}_{n=1}^{\infty} u f(x, y) \in \Lambda_{0} B V\left(T^{2}\right)$, mo $I_{r}(f) \leqslant C \Lambda_{0} V f$ при всех $r$.

Теорема 2. Пусть функиия $f(x, y) \in \Lambda_{\varepsilon} B V\left(T^{2}\right)$, әде последовательность $\Lambda_{\varepsilon}$ бълла определена в теореме $1, I_{r}(f) \rightarrow 0$ при $r \rightarrow \infty$, точка $\left(x_{0}, y_{0}\right) \in T^{2} u$ существуют четыре конечных предела $\lim _{x \rightarrow x_{0}, y \rightarrow y_{0}} f(x, y)$ по соответствующим координатным квадрантам. Тогда ряд Фурье функции $f(x, y)$ сферически сходится в точке $\left(x_{0}, y_{0}\right)$ к среднему арифметическому этих пределов.

Ранее, в статье [5] автором было показано, что указанные в теореме 2 пределы существуют в любой точке, если $f(x, y) \in \Lambda B V\left(T^{2}\right)$, где

$$
\sum_{j=1}^{\infty} \lambda_{j}^{-2}=\infty
$$


Заметим, что из одного результата Ш. А. Алимова и В. А. Ильина [7], которые установили, что для любого $\varepsilon>0$ найдется функция $f(x, y) \in \operatorname{Lip}\left(\frac{1}{2}-\varepsilon\right)$ с неограниченными сферическими частичными суммами, вытекает, что в теореме 1 нельзя вместо $\Lambda_{\varepsilon}$ взять какую-либо последовательность вида $\left\{n^{3 / 4+\varepsilon}\right\}_{n=1}^{\infty}$ с $\varepsilon>0$. Вопрос о нахождении наиболее широкого класса $\Lambda B V\left(T^{2}\right)$, для которого верно утверждение теоремы 1 , остается пока открытым. Неясно также, не вытекает ли условие (2) из того, что $f(x, y) \in \Lambda_{\varepsilon} B V\left(T^{2}\right)$. Представляется весьма вероятньм, что дело обстоит именно так. Отметим, также, что метод доказательства теорем 1 и 2 позволяет получить и соответствующий принцип локализации для сферических частичных сумм двойных рядов Фурье функций из классов Ватермана.

В четвертом параграфе обсуждается вопрос о поведении сферических частичных сумм рядов Фурье характеристических функций выпуклых множеств $A \subset T^{2}$. Ранее автором [8] изучалась аналогичная задача для прямоугольных частичных сумм и были, в частности, получены такие результаты.

ТЕОРема А. Пусть выпуклое замкнутое невырожденное множество $A \subset(-\pi, \pi)^{2}$. Тогда двойной тригонометрический ряд Фурье характеристической функиии $X_{A}(x, y)$ сходится по Прингсхейму $\kappa 1$ в любой внутренней точке $A, \kappa$ нулю в любой точке множества $T^{2} \backslash A$ и $\frac{1}{2}$ в каждой точке границы $\delta A$, в которой существует касательная $к \delta A$, не параллельная ни одной из осей координат. Сходимость по кубам имеет место в каждой точке.

ТЕОРема Б. Пусть выпуклое замкнутое невырожденное мнолсество $A \subset(-\pi, \pi)^{2}, \quad$ и его граница имеет касательную в каждой точке. Тогда множество расходимости по Прингсхейму двойного ряда Фурье характеристической функции $X_{A}(x, y)$ состоит на $T^{2}$ не более, чем из восьми, и не менее, чем из четырех точек (все возможности реализуются).

Основным результатом четвертого параграфа является нижеследующий.

ТЕОРема 3. Пусть выпуклое замкнутое невырожденное множество $A \subset(-\pi, \pi)^{2}$. Тогда двойной тригонометрический ряд Фурве характеристической функции $X_{A}(x, y)$ сходится по кругам всюду на $T^{2}$. Точнее, он сходится $\kappa 1$ в любой внутренней точке $A$ и к нулю на $T^{2} \backslash A$. Далее, если в точке $\left(x_{0}, y_{0}\right) \in \delta A$ односторонние касательнье $\kappa \delta A$ образуют угол $\alpha \in(0, \pi]$, то $S_{R}\left(X_{A}, x_{0}, y_{0}\right) \rightarrow \alpha /(2 \pi)$ при $R \rightarrow \infty$. B частности, в любой точке границь, где есть касательная, ряд Фурье сферически сходится $\kappa \frac{1}{2}$.

Там же будет показано, что уже в трехмерном случае подобный результат неверен. Так, сферические частичные суммы ряда Фурье характеристической функции трехмерного единичного шара не сходятся в начале координат, а в пространствах большей размерности аналогичные величины вообще не являются ограниченными (см. [4]). 


\section{§2. Вспомогательные результаты}

Пусть, как и во введении, при $R \geqslant 1$ ядро

$$
D_{R}^{\prime}(x, y)=\frac{1}{4} \sum_{m, n \in \mathbb{Z}} \alpha_{m, n} e^{-i(m x+n y)},
$$

где $\alpha_{m, n}=1$, если $I_{m, n} \subset B_{R}$, и $\alpha_{m, n}=\frac{\xi\left(I_{m, n} \cap B_{R}\right)}{\xi\left(I_{m, n}\right)}$ в противном случае, а

$$
\xi(E)=\iint_{E} e^{-i t x} e^{-i s y} d t d s .
$$

Отметим, что

$$
\begin{aligned}
\xi\left(I_{m, n}\right) & =\int_{m-\frac{1}{2}}^{m+\frac{1}{2}} e^{-i t x} d t \int_{n-\frac{1}{2}}^{n+\frac{1}{2}} e^{-i s y} d s \\
& =\frac{4 e^{-i m x} e^{-i n y} \sin (x / 2) \sin (y / 2)}{x y}=\frac{e^{-i m x} e^{-i n y}}{w(x) w(y)}
\end{aligned}
$$

где

$$
w(\tau)=\frac{\tau}{2 \sin (\tau / 2)}
$$

Поэтому

$$
\begin{aligned}
4 D_{R}^{\prime}(x, y) & =\sum_{m, n \in \mathbb{Z}} \alpha_{m, n} w(x) w(y) \xi\left(I_{m, n}\right)=w(x) w(y) \sum_{m, n \in \mathbb{Z}} \xi\left(I_{m, n} \cap B_{R}\right) \\
& =w(x) w(y) \iint_{B_{R}} e^{-i t x} e^{-i s y} d t d s \\
& =w(x) w(y) \iint_{B_{R}} e^{i(t x+s y)} d t d s=w(x) w(y) D_{R}^{\prime \prime}(x, y) .
\end{aligned}
$$

Лемма 1. Пусть $R \geqslant 1$, точка $(x, y) \in T^{2} u r=\sqrt{x^{2}+y^{2}} \geqslant 1 / R$. Тогда

$$
D_{R}^{\prime \prime}(x, y)=\sqrt{8 \pi} \frac{R^{2}}{(r R)^{3 / 2}}\left(\sin \left(R r-\frac{\pi}{4}\right)+\frac{3}{8 r R} \cos \left(R r-\frac{\pi}{4}\right)+\frac{\beta_{R}(x, y)}{(r R)^{2}}\right),
$$

причем существует такая абсолютная постоянная $C$, что $\left|\beta_{R}(x, y)\right| \leqslant C$ для всех $R, x$ и у, удовлетворяющих условию лемми.

ДокАЗАТЕЛЬСТво. Отметим, что $D_{R}^{\prime \prime}(x, y)$ является преобразованием Фурье характеристической функции шара радиуса $R$. Как известно (см. [9, с. 154]), преобразование Фурье радиальной функции является радиальной функцией. Поэтому, с учетом асимптотики функций Бесселя (см. [10, с. 222 и 229]), будем иметь

$$
\begin{aligned}
D_{R}^{\prime \prime}(x, y) & =\iint_{B_{R}} e^{i t r} d t d s=2 \int_{-R}^{R} \sqrt{R^{2}-t^{2}} e^{i t r} d t \\
& =4 R^{2} \int_{0}^{1} \sqrt{1-u^{2}} \cos (r R u) d u=\frac{2 \pi R^{2}}{R r} J_{1}(R r) \\
& =\sqrt{8 \pi} \frac{R^{2}}{(r R)^{3 / 2}}\left(\sin \left(R r-\frac{\pi}{4}\right)+\frac{3}{8 r R} \cos \left(R r-\frac{\pi}{4}\right)+O\left(\frac{1}{(R r)^{2}}\right)\right)
\end{aligned}
$$

при $R \rightarrow \infty$. Но это эквивалентно утверждению леммы. 
СлеДСТвИЕ 1 . Пусть $R \geqslant 1,(u, v) \in(0, \pi)^{2} u v \geqslant u$. Тогда

$$
\left|\int_{v}^{\pi} D_{R}^{\prime}(u, y) d y\right| \leqslant \frac{C R}{(R v)^{3 / 2}}, \quad\left|\int_{u}^{\pi} D_{R}^{\prime}(x, v) d x\right| \leqslant \frac{C R}{R^{3 / 2} u v^{1 / 2}}
$$

$u$

$$
\left|\int_{u}^{\pi} D_{R}^{\prime}(x, v) d x\right| \leqslant \frac{C R}{R v+1}
$$

ДокАЗАТЕЛЬСТво. Последняя оценка была установлена автором в статье [5]. Докажем два первых неравенства. Благодаря второй теореме о среднем достаточно доказать их для интеграла от $D_{R}^{\prime \prime}(u, v)$. Вначале установим первую из них. Обозначая $r=\sqrt{u^{2}+y^{2}}$, будем иметь

$$
\left|\int_{v}^{\pi} D_{R}^{\prime \prime}(u, y) d y\right| \leqslant C R^{2}\left(\left|\int_{v}^{\pi} \frac{\sin (R r-\pi / 4)}{(R r)^{3 / 2}} d y\right|+\int_{v}^{\pi} \frac{d y}{(R r)^{5 / 2}}\right)
$$

Интегрируя по частям, получим

$$
\begin{aligned}
&\left|\int_{v}^{\pi} \frac{\sin (R r-\pi / 4)}{(R r)^{3 / 2}} d y\right| \\
& \leqslant\left|-\int_{v}^{\pi} \frac{r}{R y(R r)^{3 / 2}} d_{y} \cos \left(R r-\frac{\pi}{4}\right)\right| \\
& \leqslant \frac{\left|\cos \left(R\left(u^{2}+v^{2}\right)^{1 / 2}-\pi / 4\right)\right|}{v R^{5 / 2}\left(u^{2}+v^{2}\right)^{1 / 4}} \\
& \quad+\frac{C}{R^{5 / 2}}+\left|\frac{1}{R^{5 / 2}} \int_{v}^{\pi} \cos \left(R r-\frac{\pi}{4}\right)\left(\frac{1}{y^{2} r^{1 / 2}}+\frac{1}{2 r^{5 / 2}}\right) d y\right| \\
& \leqslant \frac{C}{R(R v)^{3 / 2}} .
\end{aligned}
$$

В то же время, ясно, что

$$
\int_{v}^{\pi} \frac{d y}{(R r)^{5 / 2}} \leqslant \frac{1}{R^{5 / 2}} \int_{v}^{\pi} \frac{d y}{y^{5 / 2}} \leqslant \frac{C}{R(R v)^{3 / 2}}
$$

откуда и вытекает первое утверждение следствия 1.

Доказательство второго неравенства проходит по той же схеме. Здесь полагаем $r=\sqrt{v^{2}+x^{2}}$. Далее, например,

$$
\int_{u}^{\pi} \frac{d x}{(R r)^{5 / 2}} \leqslant \frac{1}{R^{5 / 2} v^{1 / 2}} \int_{u}^{\pi} \frac{d x}{x^{2}} \leqslant \frac{C}{R^{5 / 2} u v^{1 / 2}} .
$$

Аналогичные изменения вносятся и во все остальные оценки. Таким образом, следствие 1 полностью доказано. 
Лемма 2. Пусть $R \geqslant 1 u(u, v) \in(0, \pi)^{2}$. Тогда справедливъ оценки

$$
\left|\int_{u}^{\pi} \int_{v}^{\pi} D_{R}^{\prime \prime}(x, y) d x d y\right| \leqslant \frac{C}{R \max (u, v)+1}
$$

$u$

$$
\left|\int_{u}^{\pi} \int_{v}^{\pi} D_{R}^{\prime \prime}(x, y) d x d y\right| \leqslant \frac{C}{(R \max (u, v)+1)^{1 / 2}(R \min (u, v)+1)} .
$$

ДокАЗАТЕльство. Оценка (3) была установлена автором в работе [5]. Если $R u<1$ или $R v<1$, то неравенство (4) вытекает из оценки (3). Поэтому будем считать, что $R u \geqslant 1$ и $R v \geqslant 1$. Воспользовавшись леммой 1 , будем иметь

$$
\begin{aligned}
\int_{u}^{\pi} & \int_{v}^{\pi} D_{R}^{\prime \prime}(x, y) d x d y \\
= & C R^{2}\left(\int_{u}^{\pi} \int_{v}^{\pi} \frac{\sin (R r-\pi / 4)}{(r R)^{3 / 2}} d x d y\right. \\
& \left.\quad+C_{1} \int_{u}^{\pi} \int_{v}^{\pi} \frac{\cos (R r-\pi / 4)}{(r R)^{5 / 2}} d x d y+\int_{u}^{\pi} \int_{v}^{\pi} \frac{\beta_{R}(x, y)}{(r R)^{7 / 2}} d x d y\right) \\
= & C R^{2}\left(I_{1}+I_{2}+I_{3}\right) .
\end{aligned}
$$

Ясно, что

$$
\left|I_{3}\right| \leqslant C R^{-2} \int_{u R}^{\pi R} \int_{v R}^{\pi R} \frac{d \tau d \xi}{\left(\tau^{2}+\xi^{2}\right)^{7 / 4}}=C R^{-2} \int_{\varphi_{1}}^{\varphi_{2}} \int_{\rho_{1}(\varphi)}^{\rho_{2}(\varphi)} \frac{\rho d \rho d \varphi}{\rho^{7 / 2}}
$$

где $0<\varphi_{1}<\pi / 4<\varphi_{2}<\pi / 4$ и при любом $\varphi \in\left[\varphi_{1}, \varphi_{2}\right]$ имеем $\rho_{2}(\varphi) \geqslant \rho_{1}(\varphi) \geqslant$ $R \sqrt{u^{2}+v^{2}}$. Поэтому

$$
\left|I_{3}\right| \leqslant C R^{-2} \frac{\pi}{2} \int_{R \sqrt{u^{2}+v^{2}}}^{\infty} \frac{d \rho}{\rho^{5 / 2}} \leqslant \frac{C}{R^{2}(R \max (u, v)+1)^{3 / 2}}
$$

Далее, используя вторую теорему о среднем, получим

$$
\begin{aligned}
\frac{1}{C_{1}}\left|I_{2}\right| & =\left|\int_{u}^{\pi} \int_{v}^{\pi} \frac{\cos (R r-\pi / 4)}{(r R)^{5 / 2}} d x d y\right| \\
& \leqslant R^{-2} \int_{\varphi_{1}}^{\varphi_{2}}\left|\int_{\rho_{1}(\varphi)}^{\rho_{2}(\varphi)} \frac{\rho \cos (\rho-\pi / 4)}{\rho^{5 / 2}} d \rho\right| d \varphi \\
& \leqslant \frac{\pi}{2} R^{-2} \max _{\varphi \in\left[\varphi_{1}, \varphi_{2}\right]}\left|\int_{\rho_{1}(\varphi)}^{\rho_{2}(\varphi)} \frac{\rho \cos (\rho-\pi / 4)}{\rho^{5 / 2}} d \rho\right| \\
& \leqslant C R^{-2} \frac{1}{\left(R \sqrt{u^{2}+v^{2}}\right)^{3 / 2}} \leqslant \frac{C}{R^{2}(R \max (u, v)+1)^{3 / 2}} .
\end{aligned}
$$


Затем, интегрируя по частям, установим, что

$$
\begin{aligned}
I_{1}= & \int_{u}^{\pi} \int_{v}^{\pi} \frac{\sin (R r-\pi / 4)}{(r R)^{3 / 2}} d x d y=R^{-2} \int_{\varphi_{1}}^{\varphi_{2}} \int_{\rho_{1}(\varphi)}^{\rho_{2}(\varphi)} \frac{\sin (\rho-\pi / 4)}{\rho^{1 / 2}} d \rho d \varphi \\
= & R^{-2}\left(\int_{\varphi_{1}}^{\varphi_{2}}\left(\frac{\cos \left(\rho_{1}(\varphi)-\pi / 4\right)}{\rho_{1}(\varphi)^{1 / 2}}-\frac{\cos \left(\rho_{2}(\varphi)-\pi / 4\right)}{\rho_{2}(\varphi)^{1 / 2}}\right) d \varphi\right. \\
& \left.\quad-\frac{1}{2} \int_{\varphi_{1}}^{\varphi_{2}} \int_{\rho_{1}(\varphi)}^{\rho_{2}(\varphi)} \frac{\cos (\rho-\pi / 4)}{\rho^{3 / 2}} d \rho d \varphi\right) \\
= & R^{-2}\left(I_{1,1}-I_{1,2}\right) .
\end{aligned}
$$

Аналогично оценке (7),

$$
\left|I_{1,2}\right| \leqslant \frac{C}{R^{2}(R \max (u, v)+1)^{3 / 2}}
$$

Для изучения величины $I_{1,1}$ введем следуюшие параметры. Пусть $\varphi_{0}=\operatorname{arctg}(v / u)$ и $\rho_{0}=R \sqrt{u^{2}+v^{2}}$. Заметим, что при $\varphi_{1} \leqslant \varphi \leqslant \varphi_{0}$ имеем $\rho_{1}(\varphi)=\frac{\rho_{0} \sin \varphi_{0}}{\sin \varphi}$, а при $\varphi_{0} \leqslant \varphi \leqslant \varphi_{2}$ имеем $\rho_{1}(\varphi)=\frac{\rho_{0} \cos \varphi_{0}}{\cos \varphi}$. Далее, при $\varphi_{1} \leqslant \varphi \leqslant \pi / 4$ величина $\rho_{2}(\varphi)=\frac{R \pi}{\cos \varphi}$, а при $\pi / 4 \leqslant \varphi \leqslant \varphi_{2}$ параметр $\rho_{2}(\varphi)=\frac{R \pi}{\sin \varphi}$.

Ниже, для определенности, будем считать, что $\pi / 4 \leqslant \varphi_{0}<\pi / 2$ (т.е., что $v \geqslant u$ ). Случай $0<\varphi_{0}<\pi / 4$ рассматривается совершенно аналогично. Тогда

$$
\begin{aligned}
I_{1,1}= & \int_{\varphi_{1}}^{\varphi_{0}} \frac{\cos \left(\rho_{0} \frac{\sin \varphi_{0}}{\sin \varphi}-\frac{\pi}{4}\right)}{\left(\frac{\rho_{0} \sin \varphi_{0}}{\sin \varphi}\right)^{1 / 2}} d \varphi+\int_{\varphi_{0}}^{\varphi_{2}} \frac{\cos \left(\rho_{0} \frac{\cos \varphi_{0}}{\cos \varphi}-\frac{\pi}{4}\right)}{\left(\frac{\rho_{0} \cos \varphi_{0}}{\cos \varphi}\right)^{1 / 2}} d \varphi \\
& -\int_{\varphi_{1}}^{\pi / 4} \frac{\cos \left(\frac{R \pi}{\cos \varphi}-\frac{\pi}{4}\right)}{\left(\frac{R \pi}{\cos \varphi}\right)^{1 / 2}} d \varphi-\int_{\pi / 4}^{\varphi_{2}} \frac{\cos \left(\frac{R \pi}{\sin \varphi}-\frac{\pi}{4}\right)}{\left(\frac{R \pi}{\sin \varphi}\right)^{1 / 2}} d \varphi \\
= & S_{1}+S_{2}-S_{3}-S_{4} .
\end{aligned}
$$

Отметим, что по предположению $\varphi_{0} \in[\pi / 4, \pi / 2)$, a, следовательно, $0<\cos \varphi_{0} \leqslant$ $\sqrt{2} / 2$. Делая замену переменной

$$
\theta=\frac{\cos \varphi_{0}}{\cos \varphi}, \quad d \theta=\frac{\cos \varphi_{0} \sin \varphi}{\cos ^{2} \varphi} d \varphi=\frac{\theta}{\cos \varphi_{0}} \sqrt{\theta^{2}-\cos ^{2} \varphi_{0}} d \varphi,
$$

и применяя вторую теорему о среднем, получим

$$
\left|S_{2}\right|=\frac{\cos \varphi_{0}}{\rho_{0}^{1 / 2}}\left|\int_{1}^{b} \frac{\cos \left(\rho_{0} \theta-\pi / 4\right)}{\theta^{3 / 2} \sqrt{\theta^{2}-\cos ^{2} \varphi_{0}}} d \theta\right| \leqslant \frac{C}{\rho_{0}^{3 / 2}},
$$

где число $b>1$.

Делая замену переменной

$$
\theta=\frac{\pi}{\cos \varphi}, \quad d \theta=\frac{\theta}{\pi} \sqrt{\theta^{2}-\pi^{2}} d \varphi,
$$


получим, что

$$
S_{3}=\frac{\pi}{R^{1 / 2}} \int_{\pi / \cos \varphi_{1}}^{\pi \sqrt{2}} \frac{\cos (R \theta-\pi / 4)}{\theta^{3 / 2} \sqrt{\theta^{2}-\pi^{2}}} d \theta
$$

Учитывая, что $\cos \varphi_{1}=\pi / \sqrt{\pi^{2}+v^{2}}$, при $\theta \in\left[\pi / \cos \varphi_{1}, \pi \sqrt{2}\right]$ имеем

$$
\sqrt{\theta^{2}-\pi^{2}} \geqslant \sqrt{\pi^{2}+v^{2}-\pi^{2}}=v
$$

Поэтому по второй теореме о среднем получаем

$$
\left|S_{3}\right| \leqslant \frac{C}{R^{3 / 2} v} \leqslant \frac{C}{(R v+1)^{3 / 2}}=\frac{C}{(R \max (u, v)+1)^{3 / 2}} .
$$

Аналогично предыдущей оценке устанавливается, что

$$
\left|S_{4}\right|=\frac{\pi}{R^{1 / 2}}\left|\int_{\pi / \sin \varphi_{2}}^{\pi \sqrt{2}} \frac{\cos (R \theta-\pi / 4)}{\theta^{3 / 2} \sqrt{\theta^{2}-\pi^{2}}} d \theta\right| \leqslant \frac{C}{R^{3 / 2} u},
$$

a, следовательно,

$$
\left|S_{4}\right| \leqslant \frac{C}{(R u+1)(R v+1)^{1 / 2}}=\frac{C}{(R \min (u, v)+1)(R \max (u, v)+1)^{1 / 2}} .
$$

Перейдем к оценке величины $S_{1}$. Делая замену переменной, будем иметь

$$
S_{1}=\frac{\sin \varphi_{0}}{\rho_{0}^{1 / 2}} \int_{1}^{a} \frac{\cos \left(\rho_{0} \theta-\pi / 4\right)}{\theta^{3 / 2} \sqrt{\theta^{2}-\sin ^{2} \varphi_{0}}} d \theta
$$

где

$$
a=\frac{\frac{v}{\sqrt{v^{2}+u^{2}}}}{\frac{v}{\sqrt{v^{2}+\pi^{2}}}}=\sqrt{\frac{v^{2}+\pi^{2}}{v^{2}+u^{2}}} .
$$

Применяя вторую теорему о среднем, получим, что

$$
\left|S_{1}\right| \leqslant \frac{\sin \varphi_{0}}{\rho_{0}^{1 / 2}} \frac{C}{\rho_{0} \cos \varphi_{0}} \leqslant \frac{C}{\rho_{0}^{1 / 2} R u} \leqslant \frac{C}{(R u+1)(R v+1)^{1 / 2}} .
$$

Теперь оценка (4) вытекает из формул (5)-(12), (14) и (16). Тем самым, лемма 2 полностью доказана.

ЗАмЕчАниЕ 1 . При доказательстве леммы 2 было фактически установлено (см. (5)-(13) и (15)), что в условиях леммы 2 при $u \leqslant v$ имеем

$$
\begin{aligned}
& \int_{u}^{\pi} \int_{v}^{\pi} D_{R}^{\prime \prime}(x, y) d x d y \\
& \quad=A \frac{\sin \varphi_{0}}{\rho_{0}^{1 / 2}} \int_{1}^{a} \frac{\cos \left(\rho_{0} \theta-\pi / 4\right)}{\theta^{3 / 2} \sqrt{\theta^{2}-\sin ^{2} \varphi_{0}}} d \theta+\frac{\gamma_{1}(R, u, v)}{R^{3 / 2} u}+\frac{\gamma_{2}(R, u, v)}{\rho_{0}^{3 / 2}}
\end{aligned}
$$

где $A$ - положительная абсолютная постоянная, $a=\sqrt{\frac{v^{2}+\pi^{2}}{v^{2}+u^{2}}}$ и найдется абсолютная постоянная $C$ такая, что $\left|\gamma_{1}(R, u, v)\right| \leqslant C$ и $\left|\gamma_{2}(R, u, v)\right| \leqslant C$ при всех $R \geqslant 1$ и $u, v \geqslant 1 / R$. 
Теорема В (двумерный вариант второй теоремы о среднем). Пусть $w(x, y)-$ неотрицательная монотонная в смысле Харди функция на $I=[\alpha, \beta] \times[\gamma, \delta]$, m.e.

1. функции $w(x, \gamma)$ и $w(\alpha, y)$ монотонны на соответствующих интервалах;

2. для любого прямоугольника $I^{\prime}=[a, b] \times[c, d] \subseteq I$ имеем $w\left(I^{\prime}\right) \geqslant 0$.

Тогда для любой непрерьвной функиии $f(x, y)$ на I такой, что для некоторого $C_{1}$ и любой точки $(u, v) \in I$ имеем

$$
\left|\int_{u}^{\beta} \int_{v}^{\delta} f(x, y) d x d y\right| \leqslant C_{1}
$$

въполняется оченка

$$
\left|\iint_{I} w(x, y) f(x, y) d x d y\right| \leqslant 4 C_{1} \max _{I} w(x, y)
$$

Доказательство теоремы В практически не отличается от доказательства второй теоремы о среднем в одномерном случае.

Теперь предложение 1 сразу следует из леммы 2 и теоремы В.

Перейдем к доказательству предложения 2. Вначале установим, что при предположениях и обозначениях леммы 2 имеет место равенство

$$
\begin{aligned}
Q & =\int_{1}^{a} \frac{\cos \left(\rho_{0} \theta-\pi / 4\right)}{\theta^{3 / 2} \sqrt{\theta^{2}-\sin ^{2} \varphi_{0}}} d \theta \\
& =-\frac{\sin \left(\rho_{0}-\pi / 4\right)}{\rho_{0} \cos \varphi_{0}}+\frac{\gamma_{3}(R, u, v)}{\rho_{0}}+\frac{\gamma_{4}(R, u, v)}{\rho_{0}^{2}\left(1-\sin \varphi_{0}\right)^{3 / 2}}
\end{aligned}
$$

где найдется такая постоянная $C$, что $\left|\gamma_{j}(R, u, v)\right| \leqslant C$ при $j=3,4$, любом $R \geqslant 1$ и любых $1 \geqslant v \geqslant u \geqslant 1 / R$.

Для упрошения записи положим $\beta=\sin \varphi_{0}$. Поскольку (см. доказательство леммы 2) $a=\sqrt{\frac{v^{2}+\pi^{2}}{v^{2}+u^{2}}}$, a $v \leqslant 1$, то $a \geqslant \sqrt{\frac{1+\pi^{2}}{2}}$, в то время как $\beta \leqslant 1$. Поэтому по второй теореме о среднем

$$
\left|Q-\int_{1}^{2} \frac{\cos \left(\rho_{0} \theta-\pi / 4\right)}{\theta^{3 / 2} \sqrt{\theta^{2}-\sin ^{2} \varphi_{0}}} d \theta\right| \leqslant \frac{2}{\rho_{0}}
$$

Далее, если функция

$$
\begin{gathered}
\varphi(\theta)=\left(-\frac{1}{\theta^{3 / 2}}+1\right) \frac{1}{\sqrt{\theta^{2}-\beta^{2}}}=\frac{\theta^{3 / 2}-1}{\theta^{3 / 2} \sqrt{\theta^{2}-\beta^{2}}}, \\
\text { то } 0 \leqslant \varphi(\theta) \leqslant \frac{3(\sqrt{\theta}-1)}{\sqrt{\theta-1}} \leqslant 3 \text { при } \theta \in(1,2], \text { а производная } \\
\varphi^{\prime}(\theta)=\frac{3\left(\theta^{2}-\beta^{2}\right)-2\left(\theta^{3 / 2}-1\right) \theta^{2}}{2 \theta^{5 / 2}\left(\theta^{2}-\beta^{2}\right)^{3 / 2}} \\
\geqslant \frac{3\left(\theta^{2}-1\right)-2\left(\theta^{2}-1\right) \theta^{2}}{2 \theta^{5 / 2}\left(\theta^{2}-\beta^{2}\right)^{3 / 2}} \geqslant \frac{\left(\theta^{2}-1\right)\left(3-2 \theta^{2}\right)}{2 \theta^{5 / 2}\left(\theta^{2}-\beta^{2}\right)^{3 / 2}}>0
\end{gathered}
$$


при $\theta \in(1, \sqrt{3 / 2})$. Кроме того, на отрезке $[\sqrt{3 / 2}, 2]$ функция $\varphi^{\prime}(\theta)$ ограничена. Отсюда, применяя вторую теорему о среднем и интегрируя по частям, получим

$$
\begin{aligned}
\left|\int_{1}^{2} \cos \left(\rho_{0} \theta-\frac{\pi}{4}\right) \varphi(\theta) d \theta\right| \leqslant & \left|\int_{1}^{\sqrt{3 / 2}}\right|+\left|\int_{\sqrt{3 / 2}}^{2}\right| \\
\leqslant & \max _{\theta \in[1,2]} \varphi(\theta) \frac{2}{\rho_{0}}+\frac{2}{\rho_{0}} \max _{\theta \in[1,2]} \varphi(\theta) \\
& +\frac{1}{\rho_{0}}\left|\int_{\sqrt{3 / 2}}^{2} \sin \left(\rho_{0} \theta-\frac{\pi}{4}\right) \varphi^{\prime}(\theta) d \theta\right| \leqslant \frac{C}{\rho_{0}} .
\end{aligned}
$$

Теперь рассмотрим функцию

$$
\psi(\theta)=\frac{1}{\sqrt{\theta-\beta} \sqrt{1+\beta}}-\frac{1}{\sqrt{\theta^{2}-\beta^{2}}}=\frac{\sqrt{\theta+\beta}-\sqrt{1+\beta}}{\sqrt{\theta^{2}-\beta^{2}} \sqrt{1+\beta}} .
$$

Здесь снова имеем

$$
0 \leqslant \psi(\theta) \leqslant \frac{\theta^{2}-1}{\sqrt{\theta^{2}-\beta^{2}}} \leqslant \sqrt{\theta^{2}-1} \leqslant \sqrt{3}
$$

при $\theta \in(1,2]$. Затем,

$$
\begin{aligned}
\sqrt{1+\beta} \psi^{\prime}(\theta) & =\frac{\frac{1}{2}\left(\theta^{2}-\beta^{2}\right)-\theta(\theta+\beta-\sqrt{1+\beta} \sqrt{\theta+\beta})}{\left(\theta^{2}-\beta^{2}\right)^{3 / 2} \sqrt{\theta+\beta}} \\
& =\frac{-\frac{1}{2} \theta^{2}-\frac{1}{2} \beta^{2}-\theta \beta+\theta \sqrt{1+\beta} \sqrt{\theta+\beta}}{\left(\theta^{2}-\beta^{2}\right)^{3 / 2} \sqrt{\theta+\beta}} \\
& =\frac{\theta(1+\beta)^{1 / 2}-\frac{1}{2}(\theta+\beta)^{3 / 2}}{\left(\theta^{2}-\beta^{2}\right)^{3 / 2}} .
\end{aligned}
$$

Обозначим $\tau(\theta)=\theta(1+\beta)^{1 / 2}-\frac{1}{2}(\theta+\beta)^{3 / 2}$. Так как число $\beta \in(0,1]$, то

$$
\tau(1)=(1+\beta)^{1 / 2}-\frac{1}{2}(1+\beta)^{3 / 2}=(1+\beta)^{1 / 2} \frac{1}{2}(1-\beta) \geqslant 0 .
$$

Кроме того, функция

$$
\tau^{\prime}(\theta)=(1+\beta)^{1 / 2}-\frac{3}{4}(\theta+\beta)^{1 / 2}
$$

монотонно убьвает на $[1,2]$. Поскольку

$$
\tau(2)=2(1+\beta)^{1 / 2}-\frac{1}{2}(2+\beta)^{3 / 2},
$$

a

$$
\begin{aligned}
4(1+\beta)-\frac{1}{4}(2+\beta)^{3} & =4+4 \beta-2-3 \beta-\frac{3}{2} \beta^{2}-\frac{1}{4} \beta^{3} \\
& =2+\beta-\frac{3}{2} \beta^{2}-\frac{1}{4} \beta^{3} \\
& \geqslant 2-\frac{3}{2}-\frac{1}{4}=\frac{1}{4}>0
\end{aligned}
$$


при $\beta \in[0,1]$, число $\tau(2)>0$ при всех $\beta$. Отсюда вытекает, что $\tau(\theta)>0$ при всех $\beta$, а потому функция $\psi(\theta)$ монотонно возрастает на $[1,2]$.

Снова применяя вторую теорему о среднем получаем, что

$$
\left|\int_{1}^{2} \cos \left(\rho_{0} \theta-\frac{\pi}{4}\right) \psi(\theta) d \theta\right| \leqslant \frac{2 \sqrt{3}}{\rho_{0}} .
$$

Теперь заметим, что

$$
\begin{aligned}
Y & =\int_{1}^{2} \frac{\cos \left(\rho_{0} \theta-\pi / 4\right)}{\sqrt{\theta-\beta}} d \theta=\int_{0}^{1} \frac{\cos \left(\rho_{0} \tau+\rho_{0}-\pi / 4\right)}{\sqrt{\tau+1-\beta}} d \tau \\
& =\cos \left(\rho_{0}-\frac{\pi}{4}\right) \int_{0}^{1} \frac{\cos \rho_{0} \tau}{\sqrt{\tau+1-\beta}} d \tau-\sin \left(\rho_{0}-\frac{\pi}{4}\right) \int_{0}^{1} \frac{\sin \rho_{0} \tau}{\sqrt{\tau+1-\beta}} d \tau \\
& =\cos \left(\rho_{0}-\frac{\pi}{4}\right) Y_{1}-\sin \left(\rho_{0}-\frac{\pi}{4}\right) Y_{2} .
\end{aligned}
$$

Обозначая $\lambda=1-\beta$, будем иметь

$$
\begin{aligned}
Y_{1} & =\frac{1}{\sqrt{\lambda}} \int_{0}^{1} \frac{\cos \left(\rho_{0} \lambda(\tau / \lambda)\right)}{\sqrt{(\tau / \lambda)+1}} d \tau=\sqrt{\lambda} \int_{0}^{1 / \lambda} \frac{\cos \left(\rho_{0} \lambda \xi\right)}{\sqrt{\xi+1}} d \xi \\
& =\frac{1}{\rho_{0} \sqrt{\lambda}} \int_{0}^{1 / \lambda} \frac{1}{\sqrt{\xi+1}} d \sin \left(\rho_{0} \lambda \xi\right) \\
& =\frac{1}{\rho_{0} \sqrt{\lambda}} \frac{\sin \rho_{0}}{\sqrt{(1 / \lambda)+1}}+\frac{1}{2 \rho_{0} \sqrt{\lambda}} \int_{0}^{1 / \lambda} \frac{\sin \left(\rho_{0} \lambda \xi\right)}{(\xi+1)^{3 / 2}} d \xi .
\end{aligned}
$$

Учитьвая, что по второй теореме о среднем

$$
\left|\int_{0}^{1 / \lambda} \frac{\sin \left(\rho_{0} \lambda \xi\right)}{(\xi+1)^{3 / 2}} d \xi\right| \leqslant \frac{2}{\rho_{0} \lambda}
$$

и что $0<\lambda<1$, получаем

$$
\left|Y_{1}\right| \leqslant \frac{1}{\rho_{0}}+\frac{1}{\rho_{0}^{2} \lambda^{3 / 2}} .
$$

Действуя аналогично, будем иметь

$$
Y_{2}=\frac{1}{\rho_{0} \sqrt{\lambda}}-\frac{\cos \rho_{0}}{\rho_{0} \sqrt{\lambda} \sqrt{(1 / \lambda)+1}}-\frac{1}{2 \rho_{0} \sqrt{\xi}} \int_{0}^{1 / \lambda} \frac{\cos \left(\rho_{0} \lambda \xi\right)}{(\xi+1)^{3 / 2}} d \xi,
$$

то есть

$$
Y_{2}=\frac{1}{\rho_{0} \sqrt{\lambda}}+Y_{2}^{\prime}
$$

где

$$
\left|Y_{2}^{\prime}\right| \leqslant \frac{1}{\rho_{0}}+\frac{1}{\rho_{0}^{2} \lambda^{3 / 2}}
$$

Из формул (18)-(24) вытекает, что

$$
Q=-\frac{\sin \left(\rho_{0}-\pi / 4\right)}{\rho_{0} \sqrt{1+\beta} \sqrt{1-\beta}}+Q^{\prime}=-\frac{\sin \left(\rho_{0}-\pi / 4\right)}{\rho_{0} \cos \varphi_{0}}+Q^{\prime}
$$


где

$$
\left|Q^{\prime}\right| \leqslant \frac{C}{\rho_{0}}+\frac{C}{\rho_{0}^{2}\left(1-\sin \varphi_{0}\right)^{3 / 2}} .
$$

Тем самым, равенство (17) установлено.

Далее, интегрируя по частям, будем иметь

$$
\begin{aligned}
I(R, u, v)= & 4 \int_{u}^{\pi} \int_{v}^{\pi} D_{R}^{\prime}(x, y) d x d y=\int_{u}^{\pi} \int_{v}^{\pi} w(x) w(y) D_{R}^{\prime \prime}(x, y) d x d y \\
= & -\int_{u}^{\pi} w(x) \int_{v}^{\pi} w(y) d_{y} \int_{y}^{\pi} D_{R}^{\prime \prime}(x, \xi) d \xi d x \\
= & w(v) \int_{u}^{\pi} w(x) \int_{v}^{\pi} D_{R}^{\prime \prime}(x, \xi) d \xi d x \\
& +\int_{u}^{\pi} \int_{v}^{\pi} w(x) \int_{y}^{\pi} D_{R}^{\prime \prime}(x, \xi) d \xi w^{\prime}(y) d y d x \\
= & w(u) w(v) \int_{u}^{\pi} \int_{v}^{\pi} D_{R}^{\prime \prime}(x, y) d x d y \\
& +w(v) \int_{u}^{\pi} w^{\prime}(x) \int_{x}^{\pi} \int_{v}^{\pi} D_{R}^{\prime \prime}(t, \xi) d t d \xi d x \\
& +w(u) \int_{v}^{\pi} w^{\prime}(y) \int_{u}^{\pi} \int_{y}^{\pi} D_{R}^{\prime \prime}(t, \xi) d t d \xi d y \\
& +\int_{u}^{\pi} w^{\prime}(x) \int_{v}^{\pi} w^{\prime}(y) \int_{x}^{\pi} \int_{y}^{\pi} D_{R}^{\prime \prime}(t, \xi) d t d \xi d x d y \\
= & I_{1}+I_{2}+I_{3}+I_{4} .
\end{aligned}
$$

Известно, что функция $w(x)$ бесконечно дифференцируема на отрезке $[0, \pi]$ и что $w^{\prime}(0)=0$. Поэтому для некоторого $C>0$ имеем $\left|w^{\prime}(t)\right| \leqslant C t$ при $t \in[0, \pi]$. Используя предложение 1 , получим, что

$$
\left|I_{4}\right| \leqslant \frac{C}{R^{3 / 2}} \int_{u}^{\pi} \int_{v}^{\pi} \frac{x y}{\sqrt{\max (x, y)} \min (x, y)} d x d y \leqslant \frac{C}{R^{3 / 2}} .
$$

Далее, при $v \geqslant u$ имеем

$$
\left|I_{3}\right| \leqslant \frac{C}{R^{3 / 2}} \int_{v}^{\pi} \frac{y}{u \sqrt{y}} d y \leqslant \frac{C}{R^{3 / 2} u}
$$

и

$$
\left|I_{2}\right| \leqslant \frac{C}{R^{3 / 2}}\left(\int_{u}^{v} \frac{x}{x \sqrt{v}} d x+\int_{v}^{\pi} \frac{x}{v \sqrt{x}} d x\right) \leqslant \frac{C}{R^{3 / 2} v} \leqslant \frac{C}{R^{3 / 2} u} .
$$

Возвращаясь к обозначениям равенства (17), заметим, что если $u \geqslant C / \sqrt{R}$ и $v \leqslant 1$, TO

$$
\begin{aligned}
\rho_{0}\left(1-\sin \varphi_{0}\right) & =R \sqrt{u^{2}+v^{2}}\left(1-\frac{v}{\sqrt{u^{2}+v^{2}}}\right) \\
& =R\left(\sqrt{u^{2}+v^{2}}-v\right)=\frac{R u^{2}}{\sqrt{u^{2}+v^{2}}+v} \geqslant \frac{R u^{2}}{3 v} \geqslant \frac{C^{2}}{3} .
\end{aligned}
$$


Отсюда видно, что можно зафикисировать столь большое $C_{2}$, что при $u \geqslant C_{2} / \sqrt{R}$ получим

$$
\left|\frac{\gamma_{4}(R, u, v)}{\rho_{0}^{2}\left(1-\sin \varphi_{0}\right)^{3 / 2}}\right| \leqslant \frac{1}{40 \rho_{0} \cos \varphi_{0}} .
$$

Теперь возьмем $C_{5}$ настолько малым, чтобы (см. (26)-(28)) при $v \leqslant C_{5}$ мы имели бы

$$
\max _{i=2,3,4}\left|I_{i}\right| \leqslant \frac{A}{120 R^{3 / 2} u \sqrt{v}}
$$

а также (см. замечание 1$)$, чтобы

$$
\left|\frac{\gamma_{1}(R, u, v)}{R^{3 / 2} u}\right| \leqslant \frac{A}{200 R^{3 / 2} u \sqrt{v}}
$$

где $A$ - постоянная из замечания 1 . Далее, фиксируем $C_{4}$ так, чтобы при $v \geqslant C_{4} u$ вьполнялись оценки

$$
\left|\frac{\gamma_{2}(R, u, v)}{\rho_{0}^{3 / 2}}\right| \leqslant \frac{A}{200 R^{3 / 2} u \sqrt{v}}
$$

и

$$
\left|\frac{\gamma_{3}(R, u, v)}{\rho_{0}}\right| \leqslant \frac{1}{40 \rho_{0} \cos \varphi_{0}},
$$

где снова $A$ - постоянная из замечания 1 .

Наконец, если $2 C_{3} \leqslant C_{5} / C_{4}$, а константа $C_{1}$ настолько велика, что $2 C_{2} / \sqrt{C_{1}} \leqslant$ $C_{3}$, то при $R, u$ и $v$, удовлетворяющих условиям предложения 2 , получим (см. замечание $1,(17),(25)$ и (29)-(33))

$$
\begin{aligned}
\left|\int_{u}^{\pi} \int_{v}^{\pi} D_{R}^{\prime}(x, y) d x d y\right| \geqslant & \frac{A}{\rho_{0}^{1 / 2}}\left(\frac{\left|\sin \left(\rho_{0}-\pi / 4\right)\right|}{\rho_{0} \operatorname{ctg} \varphi_{0}}-\frac{2 \sin \varphi_{0}}{40 \rho_{0} \cos \varphi_{0}}\right) \\
& -\frac{2 A}{200 R^{3 / 2} u \sqrt{v}}-\frac{3 A}{120 R^{3 / 2} u \sqrt{v}} \\
\geqslant & \frac{A}{R^{3 / 2} u \sqrt{v}}\left(\frac{1}{10}-\frac{1}{20}-\frac{1}{100}-\frac{1}{40}\right) \\
= & \frac{C_{6}}{R^{3 / 2} u \sqrt{v}}
\end{aligned}
$$

Таким образом, предложение 2 установлено полностью. 


\section{§3. Поведение сферических частичных сумм рядов Фурье функций из классов Ватермана}

Доказательство теоремы 1 проведем по схеме, являющейся модификацией предложенной автором в статье [5].

Отметим, вначале, что

$$
\begin{aligned}
& \left\|S_{R-1}(f)-S_{R}^{\prime}(f)\right\|_{C\left(T^{2}\right)} \\
& \leqslant \sup _{x, y \in T} \sum_{R-1<|(m, n)| \leqslant R+1} \frac{1}{(2 \pi)^{2}} \\
& \quad \times\left|\int_{T+x} \int_{T+y} f(t, s) w(t-x) w(s-y) \iint_{B_{R} \cap I_{m, n}} e^{-i((t-x) u+(s-y) v)} d u d v d t d s\right| \\
& \leqslant \frac{1}{(2 \pi)^{2}} \sup _{x, y \in T} \iint_{[-1,1]^{2}} \sum_{R-1<|(m, n)| \leqslant R+1} \mid \int_{T} \int_{T} f(t+x, s+y) \\
& \times w(t) w(s) e^{-i(t u+s v)} e^{-i t m} e^{-i s n} d t d s \mid d u d v \\
& \leqslant C I_{R}(f),
\end{aligned}
$$

откуда видно, что теорему можно доказывать для сумм $S_{R}^{\prime}(f, x, y)$. Далее, не ограничивая общности, проведем оценки только в точке $(0,0)$, т.е. докажем, что

$$
\left|S_{R}^{\prime}(f, 0,0)\right| \leqslant C(\varepsilon) \Lambda_{\varepsilon} V f .
$$

Мы можем также предположить, что на $T^{2} f(x, y) \neq 0$ только в тех точках, где $x \geqslant 0$ и $y \geqslant 0$.

Фиксируем некоторое $R>1$. Подберем $\nu$ так, что $2^{\nu-1}<R \leqslant 2^{\nu}$. Введем в рассмотрение множества

$$
A_{0}=\left[0, \frac{\pi}{R}\right]^{2}, \quad A_{s}=\left[0, \frac{\pi}{R} 2^{s}\right] \times\left[\frac{\pi}{R} 2^{s-1}, \frac{\pi}{R} 2^{s}\right]
$$

при $s=1,2, \ldots, \nu-1$,

$$
A_{\nu}=[0, \pi] \times\left[\frac{\pi}{R} 2^{\nu-1}, \pi\right] ; \quad B_{s}=\left[\frac{\pi}{R} 2^{s-1}, \frac{\pi}{R} 2^{s}\right] \times\left[0, \frac{\pi}{R} 2^{s-1}\right]
$$

при $s=1,2, \ldots, \nu-1$ и $B_{\nu}=\left[\frac{\pi}{R} 2^{\nu-1}, \pi\right] \times\left[0, \frac{\pi}{R} 2^{\nu-1}\right]$.

Тогда

$$
\begin{aligned}
S_{R}^{\prime}(f, 0,0) & =\frac{1}{\pi^{2}}\left(\sum_{s=0}^{\nu} \iint_{A_{s}} f(x, y) D_{R}^{\prime}(x, y) d x d y+\sum_{s=1}^{\nu} \iint_{B_{s}} f(x, y) D_{R}^{\prime}(x, y) d x d y\right) \\
& =\frac{1}{\pi^{2}}\left(\sum_{s=0}^{\nu} I_{s}+\sum_{s=1}^{\nu} I_{s}^{\prime}\right) .
\end{aligned}
$$

Проведем дальнейшие оценки для $I_{s}$ (величины $I_{s}^{\prime}$ оцениваются аналогично). Фиксируем $s \in[1, \nu]$ (в дальнейшем считаем $s<\nu$, так как при $s=\nu$ лишш несколько усложняется запись). 
Введем в рассмотрение точки $x_{k}=\frac{\pi \sqrt{k} \sqrt{2^{s}}}{R}$ при $k=0,1, \ldots, 2^{s}$ и $y_{l}=\frac{\pi l}{R}$ при $l=2^{s-1}, \ldots, 2^{s}$. Пусть одномерные отрезки $\Delta_{k}=\left[x_{k-1}, x_{k}\right]$ при $k=1, \ldots, 2^{s}$ и $\Delta_{l}^{\prime}=\left[y_{l-1}, y_{l}\right]$ при $l=2^{s-1}+1, \ldots, 2^{s}$, а двумерные отрезки $\Delta_{k, l}=\Delta_{k} \times \Delta_{l}^{\prime}$. Теперь определим функцию

$$
g_{s}(x, y)=\sum_{k=1}^{2^{s}} \sum_{l=2^{s-1}+1}^{2^{s}} f\left(x_{k}, y_{l}\right) X_{\Delta_{k, l}}(x, y) .
$$

Рассмотрим вначале выражение

$$
\begin{aligned}
S_{R}^{\prime}\left(g_{s}, 0,0\right)= & \frac{1}{\pi^{2}} \sum_{k=1}^{2^{s}} \sum_{l=2^{s-1}+1}^{2^{s}} f\left(x_{k}, y_{l}\right) \iint_{\Delta_{k, l}} D_{R}^{\prime}(x, y) d x d y \\
= & \frac{1}{\pi^{2}}\left(\sum_{k=2}^{2^{s}} \sum_{l=2^{s-1}+2}^{2^{s}} f\left(\Delta_{k, l}\right) \int_{x_{k-1}}^{x_{2} s} \int_{y_{l-1}}^{y_{2} s} D_{R}^{\prime}(x, y) d x d y\right. \\
& +\sum_{k=2}^{2^{s}}\left(f\left(x_{k}, y_{2^{s-1}+1}\right)-f\left(x_{k-1}, y_{2^{s-1}+1}\right)\right) \int_{x_{k-1}}^{x_{2} s} \int_{y_{2^{s-1}}}^{y_{2} s} D_{R}^{\prime}(x, y) d y d x \\
& +\sum_{l=2^{s-1}+2}^{2^{s}}\left(f\left(x_{1}, y_{l}\right)-f\left(x_{1}, y_{l-1}\right)\right) \int_{y_{l-1}}^{y_{2^{s}}} \int_{0}^{x_{2^{s}}} D_{R}^{\prime}(x, y) d x d y \\
& \left.+f\left(x_{1}, y_{2^{s-1}+1}\right) \iint_{A_{s}} D_{R}^{\prime}(x, y) d x d y\right) \\
= & \frac{1}{\pi^{2}}\left(F_{1}+F_{2}+F_{3}+F_{4}\right) .
\end{aligned}
$$

Из предложения 1 вытекает, что для любых $k>1$ и $l$ имеем

$$
\begin{aligned}
& \left|\int_{x_{k-1}}^{x_{2} s} \int_{y_{l-1}}^{y_{2^{s}}} D_{R}^{\prime}(x, y) d x d y\right| \\
& \quad \leqslant\left|\int_{x_{k-1}}^{\pi} \int_{y_{l-1}}^{\pi}\right|+\left|\int_{x_{2} s}^{\pi} \int_{y_{l-1}}^{\pi}\right|+\left|\int_{x_{k-1}}^{\pi} \int_{y_{2 s} s}^{\pi}\right|+\left|\int_{x_{2^{s}}}^{\pi} \int_{y_{2^{s}}}^{\pi}\right| \\
& \quad \leqslant \frac{C}{R^{3 / 2} x_{k-1} \sqrt{y_{l-1}}} \leqslant \frac{C}{\sqrt{2^{s}} \sqrt{k} \sqrt{l}} \leqslant \frac{1}{s^{1+2 \varepsilon}} \frac{C}{\lambda_{k} \lambda_{l}},
\end{aligned}
$$

где $\lambda_{k}=\frac{k^{3 / 4}}{(\ln (k+1))^{1 / 2+\varepsilon}}$. Поэтому

$$
\left|F_{1}\right| \leqslant \frac{C}{s^{1+2 \varepsilon}} \sum_{k=2}^{2^{s}} \sum_{l=2^{s-1}+2}^{2^{s}} \frac{\left|f\left(\Delta_{k, l}\right)\right|}{\lambda_{k} \lambda_{l}} \leqslant \frac{C \Lambda_{\varepsilon} V f}{s^{1+2 \varepsilon}} .
$$

Далее, поскольку согласно предложению 1 имеем

$$
\left|\int_{x_{k-1}}^{x_{2} s} \int_{y_{2^{s-1}}}^{y_{2^{s}}} D_{R}^{\prime}(x, y) d x d y\right| \leqslant \frac{C}{R y_{2^{s-1}}} \leqslant \frac{C}{2^{s-1}} \leqslant \frac{C}{2^{s / 4}} \frac{1}{\lambda_{k}},
$$


TO

$$
\left|F_{2}\right| \leqslant \frac{C}{2^{s / 4}} \sum_{k=2}^{2^{s}}\left|f\left(x_{k}, y_{2^{s-1}+1}\right)-f\left(x_{k-1}, y_{2^{s-1}+1}\right)\right| \frac{1}{\lambda_{k}} \leqslant \frac{C \Lambda_{\varepsilon} V f}{2^{s / 4}} .
$$

Аналогично,

$$
\left|F_{3}\right| \leqslant \frac{C \Lambda_{\varepsilon} V f}{2^{s / 4}} .
$$

Наконец, очевидно, что

$$
\left|F_{4}\right| \leqslant \frac{C \Lambda_{\varepsilon} V f}{2^{s}} .
$$

Из оценок (35)-(39) вытекает, что

$$
\left|S_{R}^{\prime}\left(g_{s}, 0,0\right)\right| \leqslant \frac{C \Lambda_{\varepsilon} V f}{s^{1+2 \varepsilon}} .
$$

Теперь рассмотрим величину

$$
\begin{aligned}
I_{s}-S_{R}^{\prime}\left(g_{s}, 0,0\right)= & \frac{1}{\pi^{2}} \sum_{k=1}^{2^{s}} \sum_{l=2^{s-1}+1}^{2^{s}} \iint_{\Delta_{k, l}}\left(f(x, y)-f\left(x_{k}, y_{l}\right)\right) D_{R}^{\prime}(x, y) d x d y \\
= & \frac{1}{\pi^{2}}\left(\sum_{k=1}^{2^{s}} \sum_{l=2^{s-1}+1}^{2^{s}} \iint_{\Delta_{k, l}} f\left(\left(x, x_{k}\right) \times\left(y, y_{l}\right)\right) D_{R}^{\prime}(x, y) d x d y\right. \\
& +\sum_{k=1}^{2^{s}} \sum_{l=2^{s-1}+1}^{2^{s}} \iint_{\Delta_{k, l}}\left(f\left(x_{k}, y\right)-f\left(x_{k}, y_{l}\right)\right) D_{R}^{\prime}(x, y) d x d y \\
& \left.+\sum_{k=1}^{2^{s}} \sum_{l=2^{s-1}+1}^{2^{s}} \iint_{\Delta_{k, l}}\left(f\left(x, y_{l}\right)-f\left(x_{k}, y_{l}\right)\right) D_{R}^{\prime}(x, y) d x d y\right) \\
= & \frac{1}{\pi^{2}}\left(G_{1}+G_{2}+G_{3}\right) .
\end{aligned}
$$

Заметим, что из леммы 1 сразу следует, что на $A_{s}$ выполняется оценка

Поэтому

$$
\left|D_{R}^{\prime}(x, y)\right| \leqslant \frac{C R^{2}}{2^{\frac{3}{2} s}} .
$$

$$
\left|G_{1}\right| \leqslant \frac{C R^{2}}{2^{\frac{3}{2} s}} \sum_{k=1}^{2^{s}} \sum_{l=2^{s-1}+1}^{2^{s}} \iint_{\Delta_{k, l}}\left|f\left(\left(x, x_{k}\right) \times\left(y, y_{l}\right)\right)\right| d x d y .
$$

Отметим, что $\Delta_{k, l}=\Delta_{k} \times \Delta_{l}^{\prime}$, где $\Delta_{k}=\left[\frac{\pi \sqrt{2^{s}} \sqrt{k-1}}{R}, \frac{\pi \sqrt{2^{s}} \sqrt{k}}{R}\right]$. Учитывая, что $\left|\Delta_{k}\right| \leqslant \frac{C \sqrt{2^{s}}}{R \sqrt{k}}$, и обозначая через $\Delta_{1}^{\prime}=\left[0, \frac{\pi}{R}\right]$, будем иметь

$$
\begin{aligned}
& \iint_{\Delta_{k, l}}\left|f\left(\left(x, x_{k}\right) \times\left(y, y_{l}\right)\right)\right| d x d y \\
& \quad=\int_{0}^{\left|\Delta_{k}\right|} \int_{\Delta_{1}^{\prime}}\left|f\left(\left(x_{k-1}+x, x_{k}\right) \times\left(y_{l-1}+y, y_{l}\right)\right)\right| d x d y \\
& \quad \leqslant \frac{C \sqrt{2^{s}}}{\sqrt{k}} \int_{\Delta_{1}^{\prime}} \int_{\Delta_{1}^{\prime}}\left|f\left(\left(x_{k-1}+\mu_{k} x, x_{k}\right) \times\left(y_{l-1}+y, y_{l}\right)\right)\right| d x d y,
\end{aligned}
$$


где $\mu_{k}=\left|\Delta_{k}\right| /\left|\Delta_{1}^{\prime}\right|$. Поэтому (см. (42))

$$
\begin{aligned}
\left|G_{1}\right| \leqslant & \frac{C R^{2}}{2^{s}} \int_{\Delta_{1}^{\prime}} \int_{\Delta_{1}^{\prime}} \sum_{k=1}^{2^{s}} \sum_{l=2^{s-1}+1}^{2^{s}} \frac{\left|f\left(\left(x_{k-1}+\mu_{k} x, x_{k}\right) \times\left(y_{l-1}+y, y_{l}\right)\right)\right|}{\sqrt{k}} d x d y \\
\leqslant & \frac{C R^{2}}{s^{1+2 \varepsilon}} \\
& \times \sup _{(x, y) \in \Delta_{1}^{\prime} \times \Delta_{1}^{\prime}} \sum_{k=1}^{2^{s}} \sum_{l=2^{s-1}+1}^{2^{s}} \frac{\left|f\left(\left(x_{k-1}+\mu_{k} x, x_{k}\right) \times\left(y_{l-1}+y, y_{l}\right)\right)\right|}{\lambda_{k} \lambda_{l}}\left(\text { mes } \Delta_{1}^{\prime}\right)^{2} \\
\leqslant & \frac{C \Lambda_{\varepsilon} V f}{s^{1+2 \varepsilon}} .
\end{aligned}
$$

Применяя преобразование Абеля, получим

$$
\begin{aligned}
G_{2}= & \sum_{k=2}^{2^{s}} \sum_{l=2^{s-1}+1}^{2^{s}} \int_{\Delta_{l}^{\prime}} f\left(\left(x_{k-1}, x_{k}\right) \times\left(y, y_{l}\right)\right) \int_{x_{k-1}}^{x_{2} s} D_{R}^{\prime}(x, y) d x d y \\
& +\sum_{l=2^{s-1}+1}^{2^{s}} \int_{\Delta_{l}^{\prime}}\left(f\left(x_{1}, y\right)-f\left(x_{1}, y_{l}\right)\right) \int_{0}^{x_{2} s} D_{R}^{\prime}(x, y) d x d y \\
= & G_{2,1}+G_{2,2} .
\end{aligned}
$$

Отметим, что из следствия 1 вытекает, что при $(u, y) \in \Delta_{k, l}, k>1$, имеем

$$
\left|\int_{u}^{x_{2} s} D_{R}^{\prime}(x, y) d x\right| \leqslant \frac{C R}{\sqrt{k} \sqrt{2^{s}} \sqrt{l}} .
$$

Поэтому

$$
\begin{aligned}
\left|G_{2,1}\right| & \leqslant \frac{C R}{\sqrt{2^{s}}} \int_{\Delta_{1}^{\prime}} \sum_{k=2}^{2^{s}} \sum_{l=2^{s-1}+1}^{2^{s}} \frac{\left|f\left(\Delta_{k} \times\left(y_{l-1}+y, y_{l}\right)\right)\right|}{\sqrt{k} \sqrt{l}} d y \\
& \leqslant \frac{C}{s^{1+2 \varepsilon}} \sup _{y \in \Delta_{1}^{\prime}}\left(\sum_{k=2}^{2^{s}} \sum_{l=2^{s-1}+1}^{2^{s}} \frac{\left|f\left(\Delta_{k} \times\left(y_{l-1}+y, y_{l}\right)\right)\right|}{\lambda_{k} \lambda_{l}}\right) \leqslant \frac{C \Lambda_{\varepsilon} V f}{s^{1+2 \varepsilon}}
\end{aligned}
$$

и, аналогично, используя последнюю оценку следствия 1 ,

$$
\begin{aligned}
\left|G_{2,2}\right| & \leqslant C R \sup _{y \in \Delta_{1}^{\prime}}\left(\sum_{l=2^{s-1}+1}^{2^{s}} \frac{\left|f\left(x_{1}, y_{l-1}+y\right)-f\left(x_{1}, y_{l}\right)\right|}{l}\right) \int_{\Delta_{1}^{\prime}} d y \\
& \leqslant \frac{C \Lambda_{\varepsilon} V f}{2^{s / 4}} .
\end{aligned}
$$

Сходным образом,

$$
\begin{aligned}
G_{3}= & \sum_{k=1}^{2^{s}} \sum_{l=2^{s-1}+2}^{2^{s}} \int_{\Delta_{k}} f\left(\left(x, x_{k}\right) \times\left(y_{l-1}, y_{l}\right)\right) \int_{y_{l-1}}^{y_{2^{s}}} D_{R}^{\prime}(x, y) d x d y \\
& +\sum_{k=1}^{2^{s}} \int_{\Delta_{k}}\left(f\left(x, y_{2^{s-1}+1}\right)-f\left(x_{k}, y_{2^{s-1}+1}\right)\right) \int_{y_{2^{s-1}}}^{y_{2^{s}}} D_{R}^{\prime}(x, y) d x d y \\
= & G_{3,1}+G_{3,2} .
\end{aligned}
$$


Применяя следствие 1, получим, что

$$
\left|G_{3,1}\right| \leqslant \frac{C R}{2^{\frac{3}{2} s}} \sum_{k=1}^{2^{s}} \sum_{l=2^{s-1}+2}^{2^{s}} \int_{\Delta_{k}}\left|f\left(\left(x, x_{k}\right) \times\left(y_{l-1}, y_{l}\right)\right)\right| d x .
$$

Используя тот же метод, что и при оценке $G_{1}$, будем иметь

$$
\begin{aligned}
\int_{\Delta_{k}} & \left|f\left(\left(x, x_{k}\right) \times\left(y_{l-1}, y_{l}\right)\right)\right| d x \\
& =\int_{0}^{\left|\Delta_{k}\right|}\left|f\left(\left(x_{k-1}+x, x_{k}\right) \times\left(y_{l-1}, y_{l}\right)\right)\right| d x \\
& \leqslant \frac{C \sqrt{2^{s}}}{\sqrt{k}} \int_{\Delta_{1}^{\prime}}\left|f\left(\left(x_{k-1}+\mu_{k} x, x_{k}\right) \times\left(y_{l-1}, y_{l}\right)\right)\right| d x .
\end{aligned}
$$

Отсюда

$$
\begin{aligned}
\left|G_{3,1}\right| & \leqslant \frac{C R}{2^{s}} \int_{\Delta_{1}^{\prime}} \sum_{k=1}^{2^{s}} \sum_{l=2^{s-1}+2}^{2^{s}} \frac{\left|f\left(\left(x_{k-1}+\mu_{k} x, x_{k}\right) \times\left(y_{l-1}, y_{l}\right)\right)\right|}{\sqrt{k}} d x \\
& \leqslant \frac{C}{s^{1+2 \varepsilon}} \sup _{x \in \Delta_{1}^{\prime}} \sum_{k=1}^{2^{s}} \sum_{l=2^{s-1}+2}^{2^{s}} \frac{\left|f\left(\left(x_{k-1}+\mu_{k} x, x_{k}\right) \times\left(y_{l-1}, y_{l}\right)\right)\right|}{\lambda_{k} \lambda_{l}} \\
& \leqslant \frac{C \Lambda_{\varepsilon} V f}{s^{1+2 \varepsilon}} .
\end{aligned}
$$

Кроме того,

$$
\begin{aligned}
\left|G_{3,2}\right| & \leqslant \frac{C R}{2^{\frac{3}{2} s}} \sum_{k=1}^{2^{s}} \int_{\Delta_{k}}\left|f\left(x, y_{2^{s-1}+1}\right)-f\left(x_{k}, y_{2^{s-1}+1}\right)\right| d x \\
& \leqslant \frac{C R}{2^{\frac{3}{2} s}} \sum_{k=1}^{2^{s}} \sup _{x \in \Delta_{k}}\left|f\left(x, y_{2^{s-1}+1}\right)-f\left(x_{k}, y_{2^{s-1}+1}\right)\right| \frac{\sqrt{2^{s}}}{R \sqrt{k}} \\
& \leqslant \frac{C \Lambda_{\varepsilon} V f}{2^{s / 2}} .
\end{aligned}
$$

Теперь из формул (41) и (43)-(50) следует, что

$$
\left|I_{s}-S_{R}^{\prime}\left(g_{s}, 0,0\right)\right| \leqslant \frac{C \Lambda_{\varepsilon} V f}{s^{1+2 \varepsilon}}
$$

при $s \in[1, \nu]$. Поскольку неравенство

$$
\left|I_{0}\right| \leqslant C \sup _{(x, y) \in T^{2}}|f(x, y)|
$$

очевидно, а величины $I_{s}^{\prime}$ оцениваются аналогично, мы доказали, что (см. также (34) и (40))

$$
\left|S_{R}^{\prime}(f, 0,0)\right| \leqslant C(\varepsilon) \Lambda_{\varepsilon} V f
$$


а это эквивалентно основному утверждению теоремы 1. Предположим, теперь, что $f(t, s) \in \Lambda_{0} B V\left(T^{2}\right)$. Тогда, последовательно рассмотрев произведения функций, убедимся в том, что при любых $x, y \in T$ и любых $u, v \in[-1,1]$ функция $\psi_{x, y, u, v} \in \Lambda_{0} B V\left(T^{2}\right)$, причем $\Lambda_{0} V \psi_{x, y, u, v} \leqslant C \Lambda_{0} V f$. Поскольку в статье [5] для произвольной $g(t, s) \in \Lambda_{0} B V\left(T^{2}\right)$ было установлено неравенство

$$
\left|a_{m, n}(g)\right| \leqslant \frac{C \Lambda_{0} V g}{\sqrt{|m|+1} \sqrt{|n|+1}},
$$

получаем, что для функции $f(t, s)$ выполняется условие (2). Таким образом, теорема 1 полностью доказана.

ЗАмЕчАнИЕ 2. При доказательстве теоремы 1 было фактически установлено, что $\left|S_{R}^{\prime}(f, 0,0)\right| \leqslant C(\varepsilon) \Lambda_{\varepsilon} V f$. Кроме того, из доказательства видно, что если $f(x, y) \in \Lambda_{\varepsilon} B V\left(T^{2}\right)$ и для некоторого $\delta>0$ функция $f(x, y)=0$ при $(x, y) \in$ $[-\delta, \delta]^{2}$, то $S_{R}^{\prime}(f, 0,0) \rightarrow 0$ при $R \rightarrow \infty$.

Для доказательства теоремы 2 нам понадобится еще одна лемма.

ЛЕмма А. Пусть функиия $f(x, y) \in \Lambda B V\left(T^{2}\right)$, әде

$$
\sum_{j=1}^{\infty} \lambda_{j}^{-1}=\infty
$$

точка $\left(x_{0}, y_{0}\right) \in T^{2}$ и в этой точке существуют четыре предела

$$
\lim _{\substack{x \rightarrow x_{0} \pm 0, y \rightarrow y_{0} \pm 0}} f(x, y)=f\left(x_{0} \pm 0, y_{0} \pm 0\right) .
$$

Тогда $\Lambda V_{[-\delta, \delta]^{2}} f \rightarrow 0$ при $\delta \rightarrow+0$.

Эта лемма была доказана А. А. Саакяном [11] для случая $\Lambda \equiv\{n\}_{n=1}^{\infty}$. В обшем случае доказательство остается практически тем же.

Теперь установим справедливость теоремы 2. Пусть для определенности $\left(x_{0}, y_{0}\right)=(0,0)$. Прежде всего заметим, что при выполнении ее условий будем иметь

$$
\left\|S_{R}-S_{R}^{\prime}\right\|_{C\left(T^{2}\right)} \rightarrow 0
$$

при $R \rightarrow \infty$. Далее при любом $\delta>0$ функцию $f(x, y)$ можно представить в виде следуюшей суммы $f(x, y)=A+f_{1}(x, y)+f_{2}(x, y)$, где $f_{1}(x, y)=$ $(f(x, y)-A) X_{[-\delta, \delta]^{2}}(x, y)$, а $A=\frac{1}{4} \sum f( \pm 0, \pm 0)$. Отметим, что $f_{1}, f_{2} \in \Lambda_{\varepsilon} B V\left(T^{2}\right)$. Применяя лемму А, замечание 2 и хорошо известный результат о непрерьвности одномерной вариации в точках непрерывности функции, получим, что для любого $\gamma>0$ найдется такое $\delta>0$, что

$$
\left|S_{R}^{\prime}\left(f_{1}, 0,0\right)\right| \leqslant \frac{\gamma}{2}
$$

Наконец, фиксировав $\delta$ и воспользовавшись замечанием 2 , получим, что при достаточно больших $R$ выполняется оценка

$$
\left|S_{R}^{\prime}\left(f_{2}, 0,0\right)\right| \leqslant \frac{\gamma}{2}
$$

Теперь требуемый результат вытекает из формул (51)-(53).

В заключение этого параграфа укажем одно условие, достаточное для выполнения оценки (2). 
Лемма 3. Пусть функиия $f(x, y) \in L_{2}\left(T^{2}\right)$ и ее смешанный модуль непрерывности в этом пространстве удовлетворяет неравенству $\omega\left(f, \delta_{1}, \delta_{2}\right)_{2} \leqslant$ $B \delta_{1}^{1 / 4} \delta_{2}^{1 / 4}$, а частнше модули непрерьвности - неравенствам $\omega_{i}\left(f, \delta_{i}\right)_{2} \leqslant$ $B \delta_{i}^{1 / 4}$, при всех $\delta_{1}, \delta_{2}>0, i=1,2$, где $B$ - некоторая постоянная. Тогда $I_{R}(f) \leqslant C\left(B+\|f\|_{2}\right)$ nрu $R \geqslant 1$.

ДокаЗАТЕЛЬСтво. Ясно, что при всех $x, y \in T$ и при всех $u, v \in[-1,1]$ модули непрерывности функции $\psi=\psi_{x, y, u, v}$ удовлетворяют таким же неравенствам, как в условии леммы, с постоянньми $C B$. Рассмотрим величину

$$
J_{R}^{\prime}(\psi)=\sum_{\substack{m \geqslant n \geqslant 0, R<|(m, n)| \leqslant R+1}}\left|a_{m, n}(\psi)\right|
$$

поскольку другие суммы оцениваются аналогично. Предположим, что число $\nu$ таково, что $2^{\nu-1} \leqslant R+1<2^{\nu}$. Тогда

$$
J_{R}^{\prime}(\psi)=\sum_{k=0}^{\nu} \sum_{\substack{m \geqslant n \geqslant 0, R<|(m, n)| \leqslant R+1, 2^{k-1} \leqslant n<2^{k}}}\left|a_{m, n}(\psi)\right|+2\|\psi\|_{2}=2\|\psi\|_{2}+\sum_{k=0}^{\nu} Y_{k}
$$

Отметим, что при всех $k$ число слагаемых в сумме $Y_{k}$ не больше $2^{k+1}$. Поэтому

$$
Y_{k} \leqslant 2^{(k+1) / 2}\left(\sum_{\substack{m \geqslant n \geqslant 0, R<|(m, n)| \leqslant R+1, 2^{k-1} \leqslant n<2^{k}}} a_{m, n}^{2}\right)^{1 / 2} \leqslant 2^{(k+1) / 2}\left(\sum_{\substack{m \geqslant 2^{\nu-2}, n \geqslant 2^{k-1}}} a_{m, n}^{2}\right)^{1 / 2} .
$$

Используя теорему М.К. Потапова о приближении углом (см. [12]), получим, что

$$
Y_{k} \leqslant C 2^{k / 2} B 2^{-\nu / 4} 2^{-k / 4}=C B 2^{-\nu / 4} 2^{k / 4} .
$$

Поскольку $\|\psi\|_{2} \leqslant C\|f\|_{2}$, суммируя по $k$, устанавливаем справедливость леммы.

\section{§4. Ряды Фурье характеристических функций выпуклых множеств}

Вначале установим такой результат.

Лемма 4. Существует такая абсолютная постоянная $C>0$, что для любого выпуклого множества $A \subseteq T^{2}$ имеем $I_{R}\left(X_{A}\right) \leqslant C / \sqrt[3]{R}$ при $R \geqslant 1$.

ДоКАЗАТЕЛЬСтво. Не ограничивая общности, будем считать, что выпуклое $A \subset[0, \pi]^{2}$. Рассмотрим, вначале, величину

$$
J_{R}\left(X_{A}\right)=\sum_{R<|(m, n)| \leqslant R+1}\left|a_{m, n}\left(X_{A}\right)\right|
$$


Отметим, что при $n \neq 0$ имеем

$$
\begin{aligned}
4 \pi^{2}\left|a_{m, n}\left(X_{A}\right)\right| & =\left|\int_{a}^{b} \int_{\varphi_{1}(x)}^{\varphi_{2}(x)} e^{-i(m x+n y)} d y d x\right| \\
& =\frac{1}{|n|}\left|\int_{a}^{b}\left(e^{-i\left(m x+n \varphi_{1}(x)\right)}-e^{-i\left(m x+n \varphi_{2}(x)\right)}\right) d x\right| \\
& \leqslant \frac{1}{|n|}\left|\int_{a}^{b} e^{-i\left(m x+n \varphi_{1}(x)\right)} d x\right|+\frac{1}{|n|}\left|\int_{a}^{b} e^{-i\left(m x+n \varphi_{2}(x)\right)} d x\right| \\
& =a_{m, n}^{\prime}+a_{m, n}^{\prime \prime} .
\end{aligned}
$$

Ясно, что

$$
a_{m, n}^{\prime \prime} \leqslant \frac{C}{|n|}
$$

при $n \neq 0$. Заметим для дальнейшего, что функции $\varphi_{1}(x)$ и $\varphi_{2}(x)$ выпуклы (вогнуты) на отрезке $[a, b]$. Известно, что в каждой точке интервала $(a, b)$ у функции $\varphi_{2}(x)$ существует односторонняя производная, являющаяся монотонной функцией. Кроме того, $\varphi_{2}(x)$ абсолютно непрерывна на $[a, b]$. Ниже через $\varphi_{2}^{\prime}(x)$ будем обозначать левую производную $\varphi_{2}(x)$ при $x \in(a, b]$. Пусть $P_{R}=\{(m, n)$ : $R<|(m, n)| \leqslant R+1\}$. Тогда $P_{R}=K_{1} \sqcup K_{2} \sqcup K_{3} \sqcup K_{4}$, где $K_{1}=N \cap L_{1}$, $K_{2}=N \cap L_{2}, K_{3}=M \cap L_{1}$ и $K_{4}=M \cap L_{2}$, причем $N=\{(m, n):|n| \geqslant m\}$, $M=\mathbb{Z}^{2} \backslash N$,

$$
L_{1}=\left\{(m, n) \in P_{R}: \operatorname{mes}\left\{x \in T:\left|\frac{m}{n}+\varphi_{2}^{\prime}(x)\right|<\frac{1}{R^{2 / 3}}\right\}<\frac{1}{R^{1 / 3}}\right\}
$$

и $L_{2}=P_{R} \backslash L_{1}$.

Отметим, что из монотонности функции $\varphi_{2}^{\prime}(x)$ вытекает, что множество

$$
E_{m, n}=E_{m, n}(R)=\left\{x \in T:\left|\frac{m}{n}+\varphi_{2}^{\prime}(x)\right|<\frac{1}{R^{2 / 3}}\right\}=\left\{\alpha_{m, n, R}, \beta_{m, n, R}\right\} \subset[a, b]
$$

при всех $(m, n) \in P_{R}$ (точки $\alpha_{m, n, R}$ и $\beta_{m, n, R}$ могут как входить в указанное множество, так и не входить в него).

Если $(m, n) \in K_{1}$, то

$$
\begin{aligned}
I(m, n) & =\left|\int_{a}^{b} e^{-i\left(m x+n \varphi_{2}(x)\right)} d x\right| \\
& \leqslant\left|\int_{[a, b] \backslash E_{m, n}} \frac{d e^{-i\left(m x+n \varphi_{2}(x)\right)}}{m+n \varphi_{2}^{\prime}(x)}\right|+\operatorname{mes} E_{m, n} \\
& =\widetilde{I}_{m, n}+\operatorname{mes} E_{m, n} \leqslant \widetilde{I}_{m, n}+R^{-1 / 3}
\end{aligned}
$$

Далее, $[a, b] \backslash E_{m, n}=\left[a, \alpha_{m, n, R}\right\} \sqcup\left\{\beta_{m, n, R}, b\right]=[a, \alpha\} \sqcup\{\beta, b]$. Мы оценим интеграл только по первому промежутку (второй оценивается аналогично). При этом будем считать, что точки $\alpha, \beta \notin E_{m, n}$ (иначе расширим интервал с сохранением 
оценки его меры). Используя теорему об интегрировании по частям в интеграле Римана-Стилтьеса и учитьвая монотонность функции $\frac{1}{m+n \varphi_{2}^{\prime}(x)}$, получим

$$
\begin{aligned}
\widehat{I}_{m, n} & =\frac{1}{n}\left|\int_{a}^{\alpha} \frac{d e^{-i\left(m x+n \varphi_{2}(x)\right)}}{\frac{m}{n}+\varphi_{2}^{\prime}(x)}\right| \\
& \leqslant \frac{2}{n} \sup _{[a, \alpha)}\left|\frac{1}{\frac{m}{n}+\varphi_{2}^{\prime}(x)}\right|+\frac{1}{n}\left|\int_{a}^{\alpha} e^{-i\left(m x+n \varphi_{2}(x)\right)} d\left(\frac{1}{\frac{m}{n}+\varphi_{2}^{\prime}(x)}\right)\right| \\
& \leqslant \frac{2}{n} R^{2 / 3}+\frac{1}{n}\left|\frac{1}{\frac{m}{n}+\varphi_{2}^{\prime}(a)}-\frac{1}{\frac{m}{n}+\varphi_{2}^{\prime}(\alpha)}\right| \leqslant \frac{4}{n} R^{2 / 3} .
\end{aligned}
$$

Учитьвая, что $|(m, n)|>R$ и что $|m| \leqslant|n|$, имеем $|n|>\frac{\sqrt{2}}{2} R>\frac{1}{2} R$. Поэтому (см. (56))

$$
a_{m, n}^{\prime \prime} \leqslant \frac{1}{n}\left(\frac{8}{n} R^{2 / 3}+R^{-1 / 3}\right) \leqslant 34 R^{-4 / 3} .
$$

Отсюда

$$
\sum_{(m, n) \in K_{1}} a_{m, n}^{\prime \prime} \leqslant\left|K_{1}\right| \frac{34}{R^{4 / 3}} \leqslant C R^{-1 / 3} .
$$

Теперь рассмотрим множество $K_{2}$. Произвольно фиксировав $\left(m_{1}, n_{1}\right) \in K_{2}$, последовательно выберем максимальную систему точек $\left\{\left(m_{k}, n_{k}\right)\right\}_{k=1}^{l}$ так, чтобы множества $E_{m_{k}, n_{k}}$ попарно не пересекались при $1 \leqslant k \leqslant l$. Заметим, что так как $[a, b] \subseteq[-\pi, \pi]$, то $l R^{-1 / 3} \leqslant 2 \pi$, откуда $l \leqslant 10 R^{1 / 3}$.

Определим множества $K_{2}(1)=\left\{(m, n) \in K_{2}: E_{m, n} \cap E_{m_{1}, n_{1}} \neq \varnothing\right\}$ и $K_{2}(s)=$ $\left\{(m, n) \in K_{2}: E_{m, n} \cap E_{m_{s}, n_{s}} \neq \varnothing\right\} \backslash \bigcup_{r=1}^{s-1} K_{2}(r)$ при $s=2,3, \ldots$ Ясно, что $K_{2}=\bigsqcup_{s=1}^{l} K_{2}(s)$. Предположим, что $1 \leqslant s \leqslant l$ и $(m, n) \in K_{2}(s)$. Тогда найдется $t \in E_{m, n} \cap E_{m_{s}, n_{s}}$. Поэтому

$$
\left|\frac{m}{n}-\frac{m_{s}}{n_{s}}\right| \leqslant\left|\frac{m}{n}-\varphi_{2}^{\prime}(t)\right|+\left|\frac{m_{s}}{n_{s}}-\varphi_{2}^{\prime}(t)\right|<\frac{2}{R^{2 / 3}} .
$$

Отсюда вытекает, что $(m, n)$ лежит в кольцевом секторе

$$
\left\{(r, \varphi): R \leqslant r \leqslant R+1, \frac{m_{s}}{n_{s}}-\frac{2}{R^{2 / 3}}<\operatorname{ctg} \varphi<\frac{m_{s}}{n_{s}}+\frac{2}{R^{2 / 3}}\right\} .
$$

Поскольку обшее число целочисленных точек в таком секторе не больше, чем $\frac{C}{R^{2 / 3}} R=C R^{1 / 3}$, где $C$ - абсолютная постоянная, мы получаем, что

$$
\left|K_{2}(s)\right| \leqslant C R^{1 / 3} \text {. }
$$

Поэтому

$$
\left|K_{2}\right| \leqslant C R^{1 / 3} l \leqslant C R^{2 / 3} .
$$

Отсюда, учитьвая оценку (55), будем иметь

$$
\sum_{(m, n) \in K_{2}} a_{m, n}^{\prime \prime} \leqslant\left|K_{2}\right| \frac{2 \pi}{|n|} \leqslant C R^{2 / 3} \frac{10}{R}=C R^{-1 / 3}
$$


Поскольку суммы по множествам $K_{3}$ и $K_{4}$ оцениваются аналогично, из неравенств (57) и (58) вытекает, что

$$
\sum_{R<|(m, n)| \leqslant R+1} a_{m, n}^{\prime \prime} \leqslant C R^{-1 / 3}
$$

Величины $a_{m, n}^{\prime}$ оцениваются точно так же, а потому (см. (54))

$$
J_{R}\left(X_{A}\right)=\sum_{R<|(m, n)| \leqslant R+1}\left|a_{m, n}\left(X_{A}\right)\right| \leqslant C R^{-1 / 3} .
$$

Пусть $x_{l}=\frac{\pi l}{k}$ при $l=0,1, \ldots, k$ и функция

$$
w_{k}(x)=\sum_{l=1}^{k} w\left(x_{l-1}\right) X_{\left(x_{l-1}, x_{l}\right]}(x)=\sum_{l=1}^{k-1}\left(w\left(x_{l-1}\right)-w\left(x_{l}\right)\right) X_{\left(0, x_{l}\right]}+w\left(x_{k-1}\right) .
$$

Тогда, пользуясь тем, что при любом $l$ множество $A_{l}=A \cap\left(0, x_{l}\right] \times[0, \pi]$ выпукло, а функция $w(x)$ монотонна и ограничена на $[0, \pi]$, получим

$$
J_{R}\left(X_{A}(x, y) w_{k}(x)\right) \leqslant \sum_{l=1}^{k-1}\left|w\left(x_{l-1}\right)-w\left(x_{l}\right)\right| J\left(X_{A_{l}}\right)+w\left(x_{k-1}\right) J\left(X_{A}\right) \leqslant C R^{-1 / 3} .
$$

Переходя к пределу при $k \rightarrow \infty$, получим, что

$$
J_{R}\left(X_{A}(x, y) w(x)\right) \leqslant C R^{-1 / 3} .
$$

Последовательно применяя те же рассуждения, получим, что при любых $u, v \in$ $[-1,1]$ имеем

$$
J_{R}\left(X_{A}(x, y) w(x) w(y) e^{-i(u x+v y)}\right) \leqslant C R^{-1 / 3} .
$$

Поскольку аналогичное неравенство очевидно справедливо и для сдвинутой характеристической функции, лемма 4 полностью установлена.

СлЕДСТВИЕ 2. Существует такая абсолютная постоянная $C$, что если выпуклое $A \subset T^{2}$ и $R \geqslant 1$, mo

$$
\left\|S_{R}\left(X_{A}\right)\right\|_{C\left(T^{2}\right)} \leqslant C .
$$

Доказательство. Ясно, что $X_{A}(x, y) \in \Lambda_{\alpha} B V\left(T^{2}\right)$, где $\Lambda_{\alpha} \equiv\left\{n^{\alpha}\right\}_{n=1}^{\infty}$, а $\alpha>1 / 2$. Поэтому требуемое утверждение вытекает из леммы 4 и теоремы 1.

ПРЕДЛОЖЕНИЕ 3. Пусть выпуклое множество $A \subset T^{2} u R \geqslant 1$. Тогда если точка $(x, y) \in T^{2} \backslash A$ и

$$
\rho=\operatorname{dist}((x, y), A)=\inf _{(s, t) \in A_{1}} \sqrt{(x-s)^{2}+(y-t)^{2}}>0
$$

где $A_{1}$ есть множество $A, 2 \pi$-периодически продолженное по каждой переменной, то

$$
\left|S_{R}^{\prime}\left(X_{A}, x, y\right)\right| \leqslant \frac{C}{\sqrt{R \rho}}
$$

әде $C$ - абсолютная постоянная. Иными словами, для $S_{R}^{\prime}\left(X_{A}\right)$ справедлив принцип локализации. 
ДокАЗАТЕЛЬСТво. Если $R \rho \leqslant 2 \pi$, то требуемая оценка вытекает из следствия 2 и леммы 4 . Пусть $R \rho \geqslant 2 \pi$. Не ограничивая общности, будем оценивать $S_{R}^{\prime}\left(X_{A}\right)$ в точке $(0,0)$. Будем также считать $A$ замкнутым. Кроме того, поскольку пересечение $A$ с любым прямоугольником снова является выпуклым множеством, достаточно, например, установить требуемую оценку для замкнутого выпуклого $A \subseteq[0, \pi]^{2} \backslash\left[0, \frac{1}{2} \rho\right]^{2}$.

Переходя к полярньм координатам и используя лемму 1, получаем

$$
\begin{aligned}
\left|4 \pi^{2} S_{R}^{\prime}(0,0)\right|= & \left|4 \iint_{A} D_{R}^{\prime}(s, t) d s d t\right| \\
= & \left|\int_{0}^{\pi / 2} \int_{r_{1}(\varphi)}^{r_{2}(\varphi)} r D_{R}^{\prime \prime}(r \cos \varphi, r \sin \varphi) w(r \cos \varphi) w(r \sin \varphi) d r d \varphi\right| \\
\leqslant & \int_{0}^{\pi / 2}\left|\int_{r_{1}(\varphi)}^{r_{2}(\varphi)} r \frac{\sqrt{8 \pi} R^{2}}{(r R)^{3 / 2}} \sin \left(R \rho-\frac{\pi}{4}\right) w(r \cos \varphi) w(r \sin \varphi) d r\right| d \varphi \\
& +C \int_{0}^{\pi / 2} \int_{r_{1}(\varphi)}^{r_{2}(\varphi)} \frac{R^{2}}{(r R)^{5 / 2}} d r d \varphi \\
\leqslant & C\left(\int_{0}^{\pi / 2}\left|\int_{R r_{1}(\varphi)}^{R r_{1}(\varphi)} \frac{\sin (\tau-\pi / 4)}{\tau^{1 / 2}} w\left(\frac{\tau \cos \varphi}{R}\right) w\left(\frac{\tau \sin \varphi}{R}\right) d \tau\right| d \varphi\right. \\
& \left.+\int_{0}^{\pi / 2} \int_{R r_{1}(\varphi)}^{\infty} \frac{d \tau}{\tau^{3 / 2}} d \varphi\right) \\
= & C\left(I_{1}+I_{2}\right) .
\end{aligned}
$$

Так как $\operatorname{Rr}_{1}(\varphi) \geqslant R \rho$ для всех $\varphi \in[0, \pi / 2]$, ясно, что

$$
I_{2} \leqslant \frac{C}{\sqrt{R \rho}}
$$

Далее, функции $w\left(\frac{\tau \cos \varphi}{R}\right)$ и $w\left(\frac{\tau \sin \varphi}{R}\right)$ монотонны по $\tau$ при любом $\varphi$ и ограничены, а потому, используя вторую теорему о среднем, получим

$$
\begin{aligned}
\mid \int_{R r_{1}(\varphi)}^{R r_{2}(\varphi)} & \frac{\sin (\tau-\pi / 4)}{\tau^{1 / 2}} w\left(\frac{\tau \cos \varphi}{R}\right) w\left(\frac{\tau \sin \varphi}{R}\right) d \tau \mid \\
& \leqslant C \sup _{[\alpha, \beta] \subseteq\left[r_{1}(\varphi), r_{2}(\varphi)\right]}\left|\int_{R \alpha}^{R \beta} \frac{\sin (\tau-\pi / 4)}{\tau^{1 / 2}} d \tau\right| \leqslant \frac{C}{\sqrt{R r_{1}(\varphi)}} \leqslant \frac{C}{\sqrt{R \rho}},
\end{aligned}
$$

откуда

$$
I_{1} \leqslant \frac{C}{\sqrt{R \rho}} .
$$

Из оценок (59)-(61) и вытекает предложение 3.

СлЕДСТВИЕ 3. Пусть замкнутое выпуклое множество $A \subset(-\pi, \pi)^{2}$, а $A^{0}$ - его внутренность. Тогда ряд Фурье функиии $X_{A}(x, y)$ сходится по кругам $\kappa 1$ на $A^{0}$ иж 0 на $T^{2} \backslash A$. 
ДокАЗАТЕЛЬСтво. Данное утверждение вытекает из леммы 4 и предложения 3.

Пусть теперь замкнутое невырожденное множество $A \subset(-\pi, \pi)^{2}$, а точка $(x, y) \in \partial A$, где $\partial A$ - граница множества $A$. Пусть, также, $l_{1}$ и $l_{2}$ - односторонние касательные к $\partial A$ в точке $(x, y)$ (их сушествование вытекает из выпуклости $A$ ).

ПРЕДЛОЖЕНИЕ 4. Пусть $\delta>0$ - это число, меньшее расстояния от $(x, y)$ до границы $T^{2}$ и $A_{\delta}$ - это выпуклое множество, являющееся пересечением круга $G_{\delta}$ с иентром в точке $(x, y)$ радиуса $\delta$ и того угла, образованного $l_{1}$ и $l_{2}$, в котором находится множество $A$. Тогда $S_{R}\left(X_{A_{\delta}}, x, y\right)-S_{R}\left(X_{A}, x, y\right) \rightarrow 0$ прu $R \rightarrow \infty$.

ДокАЗАТЕльство. В силу леммы 4 достаточно доказать, что $S_{R}^{\prime}\left(X_{A_{\delta}}, x, y\right)-$ $S_{R}^{\prime}\left(X_{A}, x, y\right) \rightarrow 0$ при $R \rightarrow \infty$. Не ограничивая обшности, будем считать, что $(x, y)=(0,0)$.

В силу предложения 3 , так как множество $A \backslash G_{\delta}$ представляется в виде разности двух выпуклых множеств, каждое из которых находится на расстоянии не меньше $\delta / 2$ от точки $(0,0)$, мы получаем, что

$$
S_{R}^{\prime}\left(X_{A \cap G_{\delta}}, 0,0\right)-S_{R}^{\prime}\left(X_{A}, 0,0\right)=S_{R}^{\prime}\left(X_{A \backslash G_{\delta}}, 0,0\right) \rightarrow 0
$$

при $R \rightarrow \infty$.

Отметим, что при любом $\gamma \leqslant \delta$ имеем $A \cap G_{\gamma} \subseteq A_{\gamma}$ и, так как $l_{1}$ и $l_{2}$ являются односторонними касательными, $\operatorname{mes}\left(A_{\gamma} \backslash\left(A \cap G_{\gamma}\right)\right)=o\left(\gamma^{2}\right)$ при $\gamma \rightarrow+0$, т.е. существует функция $\varphi(\gamma) \rightarrow 0$ при $\gamma \rightarrow+0$ такая, что $\operatorname{mes}\left(A_{\gamma} \backslash\left(A \cap G_{\gamma}\right)\right)=\gamma^{2} \varphi(\gamma)$.

Пусть нам задано некоторое $\varepsilon \in(0,1)$. Фиксируем $R_{0}$ так, чтобы выполнялись неравенства

$$
\frac{1}{R_{0} \varepsilon^{2}}<\delta \text { и } \frac{1}{\varepsilon^{4}} \varphi\left(\frac{1}{\varepsilon^{2}} t\right)<\varepsilon \text { при } t \in\left(0, \frac{1}{R_{0}}\right] .
$$

Пусть $R \geqslant R_{0}$. Положим $\gamma_{R}=\gamma=1 /\left(R \varepsilon^{2}\right)<\delta$. Тогда, так как множество $A \cap\left(G_{\delta} \backslash G_{\gamma}\right)$ представляется в виде разности двух выпуклых множеств, находящихся на расстоянии не меньше $\gamma / 2$ от точки $(0,0)$, то согласно предложению 3 имеем

$$
\sigma_{1}=\left|S_{R}^{\prime}\left(X_{A \cap\left(G_{\delta} \backslash G_{\gamma}\right)}, 0,0\right)\right| \leqslant \frac{C}{\sqrt{R \frac{1}{R \varepsilon^{2}}}}=C \varepsilon .
$$

Совершенно аналогично,

$$
\sigma_{2}=\left|S_{R}^{\prime}\left(X_{A_{\delta} \backslash A_{\gamma}}, 0,0\right)\right| \leqslant C \varepsilon
$$

Наконец,

$$
\begin{aligned}
\sigma_{3} & =\left|S_{R}^{\prime}\left(X_{A \cap G_{\gamma}}, 0,0\right)-S_{R}^{\prime}\left(X_{A_{\gamma}}, 0,0\right)\right| \\
& \leqslant \max _{(s, t) \in T^{2}}\left|D_{R}^{\prime}(s, t)\right| \operatorname{mes}\left(A_{\gamma} \backslash\left(A \cap G_{\gamma}\right)\right) \leqslant C R^{2} \gamma^{2} \varphi(\gamma) \\
& =C R^{2} \frac{1}{R^{2} \varepsilon^{4}} \varphi\left(\frac{1}{R \varepsilon^{2}}\right)<C \varepsilon .
\end{aligned}
$$


Поскольку

$$
\left|S_{R}^{\prime}\left(X_{A \cap G_{\delta}}, 0,0\right)-S_{R}^{\prime}\left(X_{A_{\delta}}, 0,0\right)\right| \leqslant \sigma_{1}+\sigma_{2}+\sigma_{3},
$$

из оценок (62)-(65) вытекает предложение 4.

Перейдем теперь к доказательству теоремы 3. Первая ее часть составляет утверждение уже доказанного следствия 3. Предположим, теперь, что $(x, y) \in \partial A$ и односторонние касательные $l_{1}$ и $l_{2}$ к $\partial A$ в точке $(x, y)$ образуют угол $\alpha \leqslant \pi$. Не ограничивая общности, будем считать, что $(x, y)=(0,0)$.

Если $A_{1}$ - это множество, ограниченное $l_{1}, l_{2}$ и единичной окружностью, то согласно лемме 4 и предложениям 3 и 4

$$
S_{R}\left(X_{A}, 0,0\right)-S_{R}^{\prime}\left(X_{A_{1}}, 0,0\right) \rightarrow 0
$$

при $R \rightarrow \infty$. Положим

$$
S_{R}^{\prime \prime}\left(X_{A_{1}}, 0,0\right)=\frac{1}{(2 \pi)^{2}} \iint_{A_{1}} D_{R}^{\prime \prime}(x, y) d x d y
$$

Докажем, что

$$
S_{R}^{\prime}\left(X_{A_{1}}, 0,0\right)-S_{R}^{\prime \prime}\left(X_{A_{1}}, 0,0\right) \rightarrow 0
$$

при $R \rightarrow \infty$.

При $R>1$ имеем

$$
\begin{aligned}
& 4 \pi^{2}\left|S_{R}^{\prime}\left(X_{A_{1}}, 0,0\right)-S_{R}^{\prime \prime}\left(X_{A_{1}}, 0,0\right)\right| \\
& =\left|\iint_{A_{1}}(w(x) w(y)-1) D_{R}^{\prime \prime}(x, y) d x d y\right| \\
& \leqslant \iint_{A_{1} \cap\left[-R^{-3 / 4}, R^{-3 / 4}\right]^{2}}|w(x) w(y)-1|\left|D_{R}^{\prime \prime}(x, y)\right| d x d y \\
& +\left|\iint_{A_{1} \backslash\left[-R^{-3 / 4}, R^{-3 / 4}\right]^{2}} D_{R}^{\prime}(x, y) d x d y\right|+\left|\iint_{A_{1} \backslash\left[-R^{-3 / 4}, R^{-3 / 4}\right]^{2}} D_{R}^{\prime \prime}(x, y) d x d y\right| \\
& =J_{1}+J_{2}+J_{3} .
\end{aligned}
$$

Поскольку множество $A_{1} \backslash\left[-R^{-3 / 4}, R^{-3 / 4}\right]^{2}$ можно разбить не более, чем на два выпуклых множества, находящихся на расстоянии не менњшем, чем $\frac{1}{2} R^{-3 / 4}$ от точки $(0,0)$, согласно предложению 3 имеем

$$
J_{2} \leqslant \frac{C}{\sqrt{R R^{-3 / 4}}}=C R^{-1 / 8}
$$

Из доказательства предложения 3 видно, что и

$$
J_{3} \leqslant C R^{-1 / 8} \text {. }
$$

Наконец, так как $|w(x) w(y)-1| \leqslant C R^{-3 / 2}$ при $(x, y) \in\left[0, R^{-3 / 4}\right]^{2}$, мы получаем, что

$$
J_{1} \leqslant C R^{-3 / 2} \max _{(x, y) \in T^{2}}\left|D_{R}^{\prime \prime}(x, y)\right| R^{-3 / 2} \leqslant \frac{C}{R} .
$$


Из оценок (68)-(71) вытекает формула (67).

Как было отмечено при доказательстве леммы 1 , функция $\frac{1}{(2 \pi)^{2}} D_{R}^{\prime \prime}(x, y)$ зависит только от $r=|(x, y)|$. Обозначим ее через $\psi_{R}(r)$. Тогда, переходя к полярным координатам, получим

$$
S_{R}^{\prime \prime}\left(X_{A_{1}}, 0,0\right)=\int_{\varphi_{0}}^{\varphi_{0}+\alpha} \int_{0}^{1} r \psi_{R}(r) d r d \varphi=\alpha \int_{0}^{1} r \psi_{R}(r) d r
$$

Предположим, теперь, что $\alpha=2 \pi / n$, где $n$ - натуральное. Пусть $A_{s}$ при $s=2,3, \ldots, n$ - это множество $A_{1}$, повернутое на угол $(s-1) \alpha$. Тогда согласно формуле (72) при $2 \leqslant s \leqslant n$ имеем

$$
S_{R}^{\prime \prime}\left(X_{A_{s}}, 0,0\right)=\alpha \int_{0}^{1} r \psi_{R}(r) d r=S_{R}^{\prime \prime}\left(X_{A_{1}}, 0,0\right) .
$$

Отсюда, учитывая, что $n \alpha=2 \pi$ и что множества $A_{k}$ и $A_{j}$ при $k \neq j$ не имеют общих внутренних точек, получаем, что

$$
n S_{R}^{\prime \prime}\left(X_{A_{1}}, 0,0\right)=\sum_{s=1}^{n} S_{R}^{\prime \prime}\left(X_{A_{s}}, 0,0\right)=S_{R}^{\prime \prime}\left(X_{B}, 0,0\right),
$$

где $B$ - единичный круг. Используя (67) и предложение 3, устанавливаем, что

$$
S_{R}^{\prime \prime}\left(X_{B}, 0,0\right) \rightarrow 1=S_{R}(1,0,0)
$$

при $R \rightarrow \infty$. Поэтому (см. (73))

$$
S_{R}^{\prime \prime}\left(X_{A_{1}}, 0,0\right) \rightarrow \frac{1}{n}=\frac{\alpha}{2 \pi}
$$

при $R \rightarrow \infty$. Таким образом (см. (66) и (67)), утверждение теоремы 3 доказано для $\alpha=2 \pi / n$. Очевидным образом, оно остается справедливым и для $\alpha=2 \pi m / n$, где числа $m$ и $n$ - натуральные.

Для завершения доказательства отметим, что фиксируя $\alpha=\pi / 2$ и используя формулы (67), (72), следствие 2 и лемму 4 , убеждаемся в сушествовании абсолютной постоянной $C$ такой, что для всех $R>1$ имеем

$$
\left|\int_{0}^{1} r \psi_{R}(r) d r\right| \leqslant C .
$$

Поэтому, если $\varepsilon>0$ и $\alpha \in(0, \varepsilon)$, то (см. (72))

$$
\left|S_{R}^{\prime \prime}\left(X_{A_{1}}, 0,0\right)\right| \leqslant C \varepsilon
$$

Отсюда и из доказанного выше вытекает утверждение теоремы 3 для произвольного $\alpha$.

В заключение приведем пример, показывающий, что в трехмерном случае дело обстоит иначе, чем в двумерном. Пусть $B$ - это единичный шар в трехмерном 
пространстве, $(m, n, k) \in \mathbb{Z}^{3}$ и $r=|(m, n, k)| \neq 0$. Тогда, используя радиальность преобразования Фурье, получим, что

$$
\begin{aligned}
8 \pi^{3} a_{m, n, k}\left(X_{B}\right) & =\iiint_{B} e^{-i(m x+n y+k z)} d x d y d z \\
& =\int_{-1}^{1} e^{-i r x} \iint_{B_{x}} d y d z d x=\pi \int_{-1}^{1} e^{-i r x}\left(1-x^{2}\right) d x \\
& =2 \pi \int_{0}^{1}\left(1-x^{2}\right) \cos r x d x=\frac{4 \pi}{r} \int_{0}^{1} x \sin r x d x \\
& =-\frac{4 \pi}{r^{2}} \cos r+\frac{4 \pi}{r^{2}} \int_{0}^{1} \cos r x d x=-\frac{4 \pi}{r^{2}} \cos r+\frac{4 \pi}{r^{3}} \sin r .
\end{aligned}
$$

Отсюда видно, что внутри шаровых слоев $2 \pi n-\pi / 4 \leqslant r \leqslant 2 \pi n+\pi / 4$, где $n=1,2, \ldots$, коэффициенты $a_{m, n, k}\left(X_{B}\right) \leqslant-C / n^{2}$, в то время, как число точек в таком слое $\geqslant C n^{2}$. Поэтому ряд Фурье для $X_{B}(x, y, z)$ сферически расходится в точке $(0,0,0)$.

\section{Список литературы}

1. Waterman $D$. On convergence of Fourier series of functions of bounded generalized variation // Studia Math. 1972. V. 44. №1. P. 107-117.

2. Chandrasekharan K., Minaksisundaram S. Some results on double Fourier series // Duke Math. J. 1947. V. 14. №3. P. 731-753.

3. Голубов Б. И. О сходимости сферических средних Рисса кратных рядов и интегралов Фурье от функций ограниченной обобщенной вариации // Матем. сб. 1972. Т. 89. № 4 . C. $630-653$.

4. Дьяченко М. И. Некоторые проблемы теории кратных рядов Фурье // УМН. 1992. T. 47. № 5. C. $97-162$.

5. Dyachenko M.I. Waterman classes and spherical partial sums of double Fourier series // Anal. Math. 1995. V. 21. № 1. P. 3-21.

6. Юдин В. А. Поведение констант Лебега // Матем. заметки. 1975. Т. 17. № 3. С. 401-405.

7. Алимов III. А., Ильин В.А. Условия сходимости спектральных разложений, соответствующих самосопряженным расширениям эллиптических операторов. 1 // Диффференц. уравнения. 1971. Т. 7. № 4. С. 670-710.

8. Дьяченко М. И. Выпуклые множества и кратные ряды Фурье // Труды МИАН. 1991. T. 200. C. $147-156$.

9. Стейн E. M., Вейс Г. Введение в гармонический анализ на евклидовых пространствах. М.: Мир, 1974.

10. Ватсон Г. Н. Теория бесселевых функций. Ч. 1. М.: ИЛ, 1949.

11. Саакян А. А. О сходимости двойных рядов Фурье функций ограниченной гармонической вариации // Изв. АН АрмССР. Сер. матем. 1986. Т. 21. №6. С. 517-527.

12. Потапов M.K. Изучение некоторых классов функций при помощи приближения "углом" // Труды МИАН. 1972. Т. 117. С. 256-291.

Московский государственный

Поступила в редакцию

университет им. М.В. Ломоносова

14.03 .1996 